\title{
A photometric and spectroscopic study of the association LH 47 in the superbubble $\mathbf{N} 44$ in the $\mathrm{LMC}^{\star, \star \star}$
}

\author{
J.-M. Will ${ }^{1, \star \star \star}$, D.J. Bomans ${ }^{2,1, \dagger}$, and A. Dieball ${ }^{1}$ \\ 1 Sternwarte der Universität Bonn, Auf dem Hügel 71, D-53121 Bonn, Germany \\ 2 University of Illinois at Urbana-Champaign, Department of Astronomy, 1002 West Green Street, Urbana, IL 61801, U.S.A.
}

Received May 13, accepted October 10, 1996

\begin{abstract}
The OB association LH 47 inside the superbubble N 44 in the Large Magellanic Cloud (LMC) is analyzed with CCD photometry. For the bright blue stars spectra in the optical and UV have been taken. The observed $U, B$, and $V$ magnitudes and colours are converted to $T_{\text {eff }}$ and $L$. From isochrone fits to the colour magnitude diagram (CMD), we find an age of the association of about 5 to 6 Myr. The stellar population outside the shell is of similar age, so there is no direct evidence for sequential star formation.

The slope of the luminosity function $(\gamma=0.24)$ agrees with what has been found for other young associations. We compare two methods to derive the slope of the initial mass function (IMF). First we count all stars along the main sequence in the $B V$ CMD. Second we count the stars between stellar evolutionary tracks in the HertzsprungRussell diagram (HRD). Both methods give mainly the same result, i.e. $x=1.37$ and $x=1.22$, respectively. The IMF of the stars outside the shell shows a slightly steeper slope than inside the shell. The slope of the IMF is very similar to values found for other associations and open clusters as well as in the solar neighborhood, thus supporting the idea of an universal shape of the IMF. LH 47 turns out to be a well behaved young association embedded in a molecular cloud.
\end{abstract}

Key words: stars: early-type - Hertzsprung-Russell (HR) diagram — stars: luminosity function, mass

Send offprint requests to: J.-M. Will, jmwill@astro.uni-bonn.de * Based on observations collected at the European Southern Observatory, La Silla, Chile and with the International Ultraviolet Explorer satellite (IUE) operated jointly by NASA, ESA, and PPARC at the VILSPA tracking station.

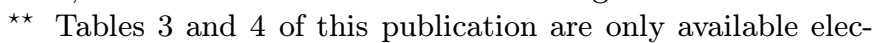
tronically, see the Editorial in A\&A 280, E1 (1993).

${ }^{\star \star \star}$ Present address: Hewlett-Packard GmbH, CTS-E/PSR, Herrenberger Str. 130, D-71034 Böblingen, Germany.

$\dagger$ Feodor Lynen-Fellow of the Alexander von HumboldtFoundation. function - open clusters and associations: individual: LH 47 - Magellanic Clouds

\section{Introduction}

The Magellanic Clouds show a large variety of bubbles, superbubbles and supergiant shells. All those prominent $\mathrm{H}$ II regions contain $\mathrm{OB}$ associations which allow us to study in detail very young stellar populations in an only moderate reddened surrounding and at a fairly well known distance. Besides the derivation of the ages of those associations, it is easy to derive the shapes of luminosity and mass functions from colour magnitude diagrams (CMDs). Due to the small ages of the associations the mass functions found from the main sequence stars are identical to their initial mass functions (IMF). In studying Large Magellanic Cloud (LMC) associations we have therefore a powerful tool for deriving the shape of the IMF over a large mass range. Furthermore, those shells are suitable laboratories to search for events of triggered star formation.

The OB association LH 47 (Lucke \& Hodge 1970) is embedded in the superbubble N 44 (Henize 1956). N 44 is also known as Shapley Constellation I (McKibben Nail \& Shapley 1953). The central shell of N 44 is identical to DEM 152 (Davies et al. 1976) and shell 1 of Meaburn \& Laspias (1991). Meaburn \& Laspias (1991) studied the kinematics of N 44 and showed that the shell is expanding with $46 \mathrm{~km} \mathrm{~s}^{-1}$ towards the observer at the front side and away from the observer with $33 \mathrm{~km} \mathrm{~s}^{-1}$ at the far side. Kontizas et al. (1996) examined the distribution of the OB stars in Shapley Constellation I and found that this complex may be embedded in a larger aggregate of stars, where they use Efremov's (1988) definition of complex and aggregate. Stasińska et al. (1986) identified a very hot O star in the H II knot N 44C with a suspected temperature of about $70000 \mathrm{~K}$. Oey \& Massey (1995) give a chart with the identifications of the different designations. 
N 44 is not only a conspicuous object in the optical and in the light of the $\mathrm{H} \alpha$ line, but also a prominent object in nearly all other wavelength ranges. N 44 can be recognized in H I (Luks \& Rohlfs 1992), in CO (Cohen et al. 1988), in ${ }^{13} \mathrm{CO}$ (Chin 1995) and also in the $\mathrm{C}_{\mathrm{II}}$ line at 158 $\mu \mathrm{m}$ (Mochizuki et al. 1994). Chin (1995) detected emission from $\mathrm{N} 44$ from the molecular transitions around $\lambda=$ $3 \mathrm{~mm}$ of $\mathrm{HC}_{2}, \mathrm{HCN}, \mathrm{HCO}^{+}, \mathrm{HNC}, \mathrm{CS}$, and $\mathrm{CN}$.

Elliot et al. (1978) published some narrow band images of $\mathrm{N} 44$. From the absence of non-thermal radio emission they concluded that supernova explosions do not play an important role in the formation of the shell. However, diffuse X-ray emission has been detected in N 44 (Chu \& MacLow 1990; Wang \& Helfand 1991) with the Einstein Observatory. Chu \& MacLow (1990) proposed that this emission is produced by supernovae when their shock fronts hit the walls of the shell. Chu et al. (1993) confirmed this scenario with pointed ROSAT observations.

The most recent CCD study of N 44 is that of Oey \& Massey (1995). They analyze the stellar population of the shell and find an age difference between the populations inside and outside the shell. They interpret this as a sign for triggered star formation. A comparison with a numerical model for the expansion of the shell, however, fails. This discrepancy can be understood, if the southern part of the X-ray bright part of N 44 is the result of a blowout structure, as proposed recently by Magnier et al. (1996).

Massey (1985) and Massey et al. (1995a,b) pointed out that for bright blue stars the $B-V$ colour is not a good temperature indicator any more and age determinations may suffer strongly from that. It is therefore essential that spectroscopic observations are performed besides wideband photometry. We thus follow here the same method as described already in Will et al. (1996) and took spectra at optical and UV wavelengths for some of the bright blue stars (see Sect. 2.2).

The structure of this paper is as follows. After the description of the data in Sect. 2, we turn to the analysis of the optical spectra (Sect. 3.1) and the UV spectra (Sect. 3.2). In Sect. 3.3 we combine these results for a final classification of those stars which have been investigated spectroscopically. The age determination of the association is described in Sect. 4. Sections 5 and 6 are devoted to the luminosity and mass functions, respectively. In Sect. 7 we finally discuss our results.

\section{Observations and data reduction}

\subsection{Photometry}

N 44 was observed with the $1.54 \mathrm{~m}$ Danish telescope on October 3rd and 4th, 1992 at La Silla with a TEK $1024 \times 1024$ CCD (ESO\#28). Table 1 gives a log of the observations. Coordinates for N 44 can be found in Table 5 (compare also Fig. 4). The linear pixel size of $24 \mu \mathrm{m}$
Table 1. Observing log for the photometry of N 44

\begin{tabular}{lcccc}
\hline Date & Filter & $\begin{array}{c}\text { Exposure } \\
\text { time }[\mathrm{sec}]\end{array}$ & $\begin{array}{c}\text { Seeing } \\
{\left[{ }^{\prime \prime}\right]}\end{array}$ & Airmass \\
\hline Oct. 3, 1992 & H $\alpha$ & 300 & 2.0 & 1.33 \\
& $I$ & 60 & 1.2 & 1.32 \\
& $I$ & 10 & 1.3 & 1.31 \\
& $I$ & 2 & 1.3 & 1.31 \\
& $R$ & 2 & 1.4 & 1.30 \\
& $R$ & 10 & 1.4 & 1.30 \\
& $R$ & 60 & 1.4 & 1.30 \\
& $V$ & 8 & 1.4 & 1.30 \\
& $V$ & 40 & 1.5 & 1.29 \\
& $V$ & 240 & 1.5 & 1.29 \\
& $B$ & 30 & 1.5 & 1.29 \\
& $B$ & 120 & 1.6 & 1.29 \\
& $B$ & 480 & 1.5 & 1.28 \\
& $B$ & 15 & 1.5 & 1.28 \\
& $U$ & 30 & 1.4 & 1.28 \\
& $U$ & 180 & 1.5 & 1.28 \\
& $U$ & 480 & 1.5 & 1.28 \\
& $I$ & 2 & 1.0 & 1.35 \\
& $R$ & 2 & 1.1 & 1.35 \\
& $R$ & 10 & 1.2 & 1.35 \\
& & 8 & 1.1 & 1.34 \\
& $B$ & 30 & 1.2 & 1.34 \\
& $U$ & 30 & 1.3 & 1.34 \\
& $U$ & 180 & 1.3 & 1.33 \\
\hline & & & &
\end{tabular}

corresponds to $0^{\prime \prime} 377$ on the sky and the resulting field of view is $6 ! 4 \times 6 ! 4$. Images have been taken in $U, B, V, R$, $I$, and $\mathrm{H} \alpha$ filters. Two examples, the $300 \mathrm{~s} \mathrm{H} \alpha$ and the $40 \mathrm{~s} V$ exposure, are shown in Fig. 1.

After standard image reduction with MIDAS and IRAF, profile fitting photometry has been performed with DAOPHOT running under IRAF. Special care has been taken to identify close optical binaries and thus to reach an exact positioning of especially the bright stars in the CMD (see also Will et al. 1995b, Sect. 4). Mean values of the photometric errors for three magnitude ranges as given by DAOPHOT are printed in Table 2 . The number of stars in the corresponding bins and the total number of stars are also given. It has to be admitted that those errors only reflect the uncertainties due to the photon statistics. Other effects, such as the quality of the profile fits and the influence of strong crowding, could increase those errors significantly.

Observing conditions on Oct. 4 were better than in the night before, thus the images of that day have first been calibrated using the calibration stars around PG $0231+$ 051 (Landolt 1992). For $U, B$, and $V$ passbands, we used the extinction coefficients from Grothues \& Gochermann (1992), i.e. $k_{U}=0.514 \mathrm{mag}, k_{B}=0.314 \mathrm{mag}$, and $k_{V}=$ $0.208 \mathrm{mag}$. For the $R$ passband we used $k_{R}=0.04 \mathrm{mag}$, for the $I$ passband we didn't apply an extinction correction. 

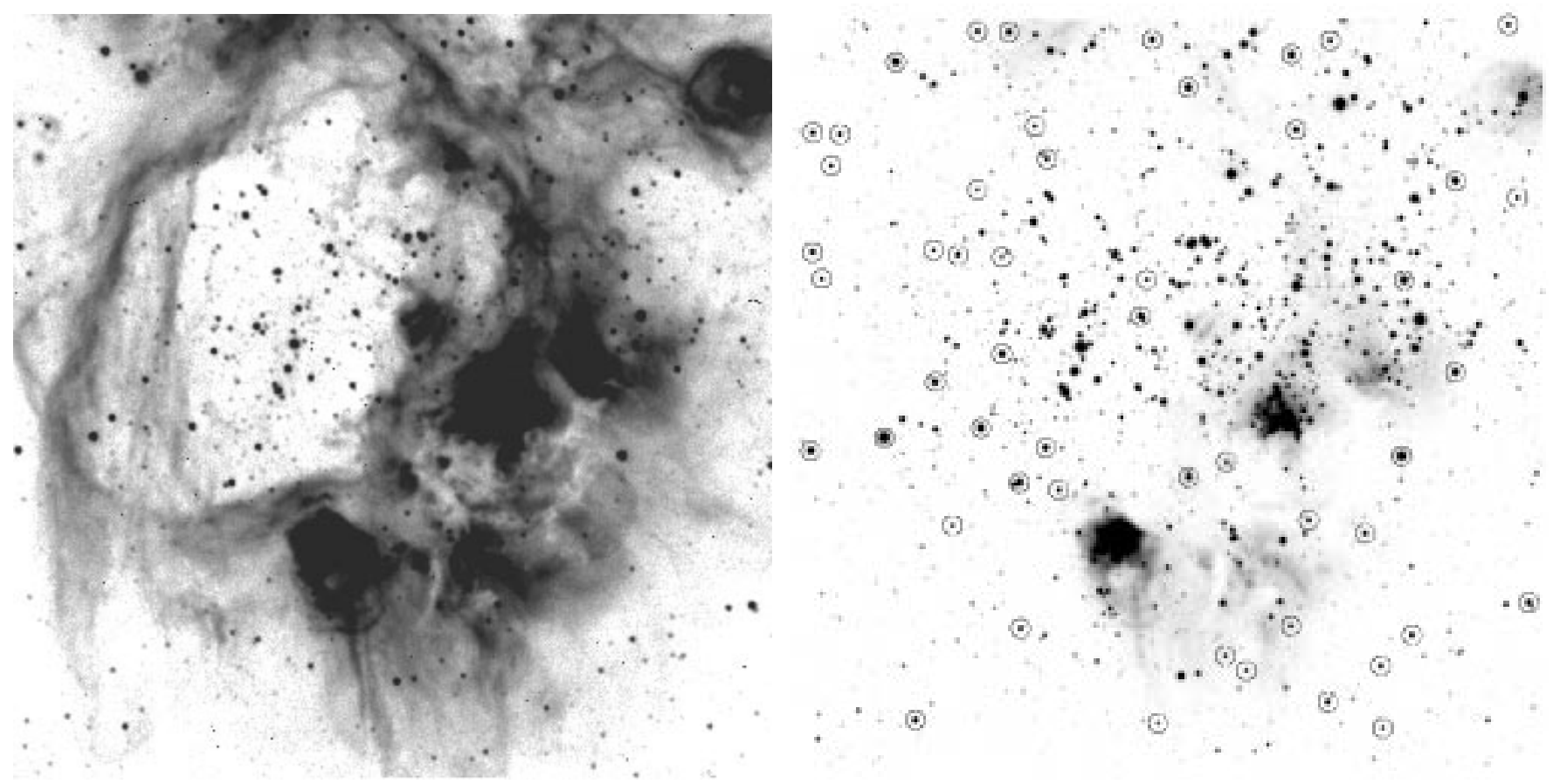

Fig. 1. CCD images of $\mathrm{N} 44$ in the $\mathrm{H} \alpha$ (left) and $V$ passband (right). North is up and east to the left. The size of the field of view is $6.4 \times 6.4$ in both cases. The stars in our field of view which are present in the MACS (Magellanic Catalogue of stars; Tucholke et al. 1996) are marked on the $V$ exposure

Table 2. Mean statistical errors of stellar magnitudes and colours. The number of stars in the corresponding magnitude range is given in parentheses. The last line gives the total number of stars in our final table

\begin{tabular}{cccccc}
\hline \multirow{2}{*}{$\begin{array}{c}\text { Magnitude } \\
\text { range [mag] }\end{array}$} & $V$ & $U-B$ & $B-V$ & $V-R$ & $V-I$ \\
\cline { 2 - 6 } & \multicolumn{5}{c}{ Mean error [mag] } \\
\hline$V<18$ & 0.009 & 0.023 & 0.013 & 0.014 & 0.016 \\
& $(547)$ & $(477)$ & $(547)$ & $(538)$ & $(529)$ \\
$18 \leq V<20$ & 0.023 & 0.119 & 0.035 & 0.044 & 0.055 \\
& $(1596)$ & $(627)$ & $(1596)$ & $(1507)$ & $(1400)$ \\
$20 \leq V$ & 0.107 & 0.490 & 0.190 & 0.168 & 0.198 \\
& $(2470)$ & $(55)$ & $(2470)$ & $(1474)$ & $(925)$ \\
\hline No. of stars: & 4613 & 1159 & 4613 & 3519 & 2854 \\
\hline
\end{tabular}

The $\mathrm{H} \alpha$ magnitudes have not been calibrated. The images of Oct. 3 then have been calibrated with those of Oct. 4 .

The final data are listed in Table 3 which is available in electronic form only (see the footnote on the front page). Table 3 contains 4613 stars for which information in $B$ and $V$ and thus also in $B-V$ is available. In addition there is a $U-B$ colour available for 1159 of these stars, a $V-R$ colour for 3519 stars, and a $V-I$ colour for 2854 stars. Figure 2 shows two CMDs ( $V$ vs. $B-V$ and $V$ vs. $U-B$ ) of all stars in the field of view.

Table 4, which is also available electronically only, gives all 51 stars which could be cross identified in the MACS (Magellanic Catalogue of Stars; Tucholke et al. 1996; de Boer et al. 1995). These stars may serve as an astrometric reference grid. A finding chart for those stars is given in Fig. 1.

The quality of the photometry and of the calibration has been checked by comparison with the photometry of Lee (1990). We could cross identify 122 stars for which $V$ and $B-V$ could be compared. For 111 of these stars also $U-B$ was available in both data sets. Figure 3 shows the differences in $V, B-V, U-B$ and the reddening free parameter $Q$ plotted against our $V$ magnitude. In $V$ a significant difference of $\overline{\Delta V} \approx-0.04 \mathrm{mag}$ is found. The differences in the colours, especially in $U-B$, are smaller. The differences are growing towards faint magnitudes since Lee's photometry is, due to the smaller telescope he used, not as deep as ours.

While our work on LH 47 was in progress Oey \& Massey (1995) published their photometry of the association. A comparison of both data sets (432 stars in common for $B$ and $V, 426$ stars for $U$ ), revealed inconsistencies with the photometry of Oey \& Massey (1995). The main sequences in their two fields (LH 47 and LH 48) do differ significantly $(\approx 0.15 \mathrm{mag})$ in $B-V$ and $U-B$ colours. This leads to approximately the same amount of deviation 


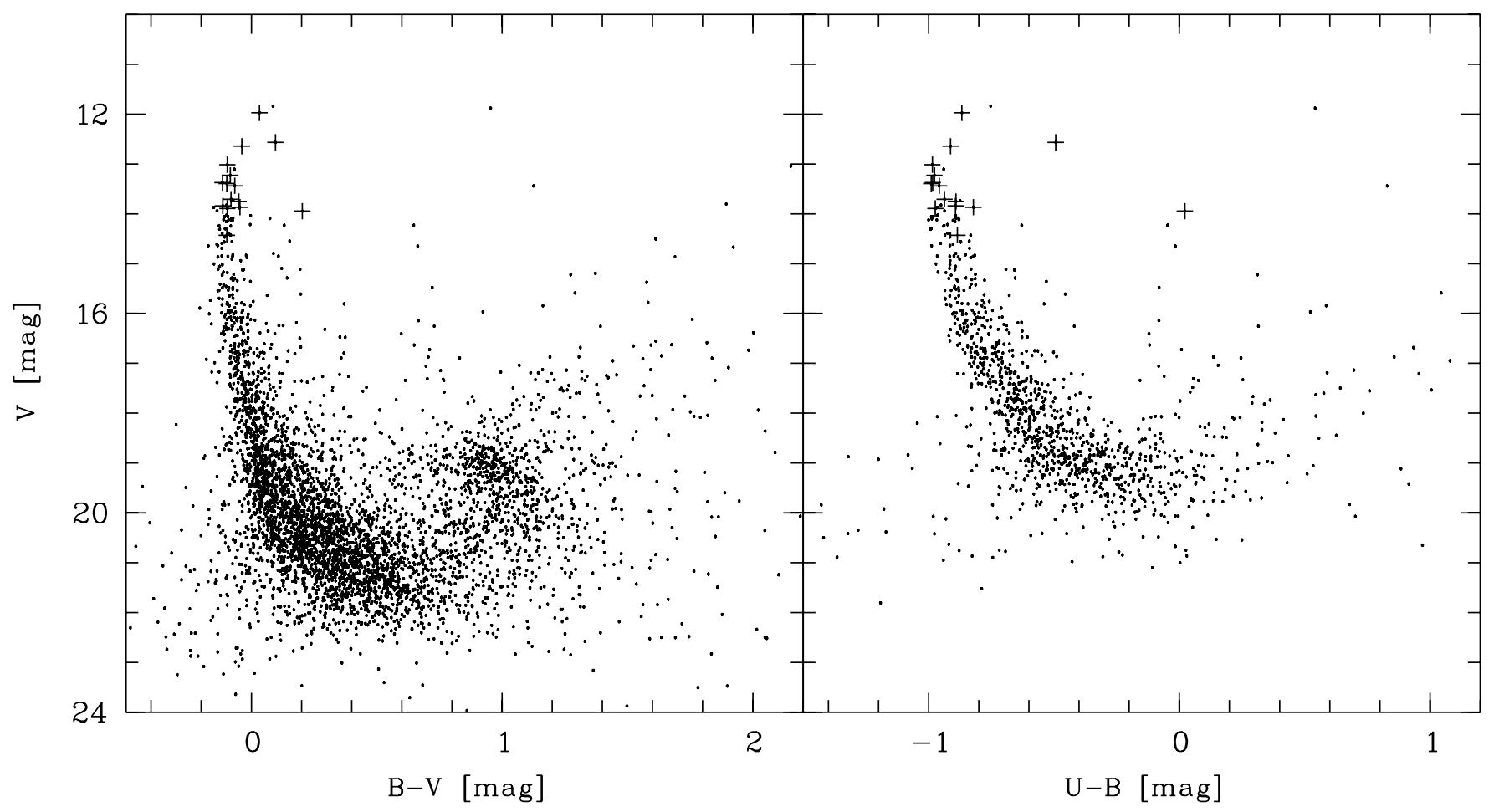

Fig. 2. CMDs, $V$ vs. $B-V$ (left panel) and $V$ vs. $U-B$ (right panel), for all stars in the field of view. The target stars for spectroscopy are marked by crosses

from our photometry (in $B-V$ for LH 47 and in $U-B$ for LH 48). With our large CCD frames we cover in one frame the main parts of the two fields of Oey \& Massey (1995). Strongly varying reddening over the area of the association cannot be found in our data. However, the problem is of that kind that the $Q$-values used by Oey \& Massey (1995) are not affected for stars brighter than $\approx 15$ th magnitude and thus their conclusions derived from their spectra together with the $Q$-values are also not affected.

\subsection{Spectroscopy}

For very hot stars the $B-V$ colour is not a good indicator for the temperature any more (see e.g. Massey 1985; Massey et al. 1995a,b; Will et al. 1996). Thus, when fitting isochrones to very young stellar populations as LH 47 with high turn off masses and no red supergiants, the age determination is quite difficult and uncertain. This is a bit better for the $U-B$ colour but there the calibration is much more difficult and unreliable. Thus we decided to take spectra of the stars at the apparent top of the main sequence with the aim to distinguish evolved and non evolved stars, i.e. to determine luminosity classes. This should allow to fix the turn off point of the main sequence and thus would result in a better determination of the age of the association.

As target stars for spectroscopy we selected bright blue stars from the top of the main sequence. They were num- bered according to decreasing brightness. Table 5 gives the coordinates and the photometric information of the target stars. Star \#14 is missing, because after selecting the stars it turned out that this star has a close neighbour and thus it has not been observed. Stars \#2 and \#9 are present in the MACS. Star \#2 is MACS J0522-679\#027 and star \#9 is MACS J0522-679\#018. The coordinates for all other stars have been calculated from the cross identification with the MACS. Figure 4 shows the target stars on a $V$ exposure of $\mathrm{N} 44$. In the CMD (Fig. 2) the target stars for spectroscopy are marked by crosses. We observed spectra of these stars in the optical wavelength range for classification purposes and in the UV for the determination of temperatures by fitting model atmospheres.

\subsubsection{Optical spectra}

Long slit spectra of the target stars in the optical wavelength range have been observed with the B\&C spectrograph at the $1.52 \mathrm{~m}$ telescope at ESO, La Silla from 24th to 26th November 1993. We used grating \#28 which gives a dispersion of $64.5 \AA / \mathrm{mm}$. The detector was a Ford CCD (ESO \#24) with $2048 \times 2048$ pixel $^{2}$ of $15 \times 15 \mu \mathrm{m}^{2}$. The slit width was $2^{\prime \prime}$ on the sky. The slit was always aligned in that way that two stars of similar brightness were observed simultaneously. The spectra cover the wavelength range from $3600 \AA$ to $5500 \AA$ with a resolution of $2.3 \AA$ (determined from the FWHM of the lines in the He-Ar 
Table 5. Target stars for spectroscopy, their coordinates, photometric information and the log of the optical and UV spectroscopy. For the optical spectra exposure times and signal-to-noise ratios are given, for the UV spectra the IUE image numbers and the exposure times are given

\begin{tabular}{|c|c|c|c|c|c|c|c|c|c|c|}
\hline \multirow[b]{2}{*}{ Star Id. } & \multicolumn{2}{|c|}{ Coordinates } & \multicolumn{4}{|c|}{ Photometry } & \multicolumn{2}{|c|}{ Optical spectra } & \multicolumn{2}{|c|}{ IUE spectra } \\
\hline & $\begin{array}{l}\alpha_{2000} \\
{\left[{ }^{\mathrm{h} \mathrm{m} \mathrm{s}}\right]}\end{array}$ & $\begin{array}{l}\delta_{2000} \\
{\left[\begin{array}{lll}0 & \prime & \prime \prime\end{array}\right]}\end{array}$ & $\begin{array}{c}V \\
{[\mathrm{mag}]}\end{array}$ & $\begin{array}{c}B-V \\
{[\mathrm{mag}]}\end{array}$ & $\begin{array}{c}U-B \\
{[\mathrm{mag}]}\end{array}$ & $\begin{array}{c}Q \\
{[\mathrm{mag}]}\end{array}$ & $\begin{array}{l}\text { Exp. } \\
\text { time }\end{array}$ & $S / N$ & $\begin{array}{l}\text { Image } \\
\text { No. }\end{array}$ & $\begin{array}{l}\text { Exp. } \\
\text { time }\end{array}$ \\
\hline N 44:01 & 52217.2 & -675657.0 & 11.97 & +0.03 & -0.87 & -0.89 & $40 \mathrm{~min}$ & 80 & $\begin{array}{l}\text { SWP } 55318 \\
\text { LWP } 31108\end{array}$ & $\begin{array}{r}12 \min \\
8 \mathrm{~min}\end{array}$ \\
\hline $\mathrm{N} 44: 02^{a}$ & 52234.9 & -675742.9 & 12.57 & +0.10 & -0.49 & -0.56 & $40 \mathrm{~min}$ & 60 & $\begin{array}{l}\text { SWP } 55319 \\
\text { LWP } 31109\end{array}$ & $\begin{array}{r}16 \mathrm{~min} \\
8 \mathrm{~min}\end{array}$ \\
\hline N 44:03 & $\begin{array}{lll}52203.8 & \end{array}$ & -675528.4 & 12.64 & -0.04 & -0.91 & -0.87 & $60 \min$ & 75 & LWP 31110 & $18 \mathrm{~min}$ \\
\hline N 44:04 & 52157.9 & -675625.1 & 13.02 & -0.10 & -0.98 & -0.92 & $60 \mathrm{~min}$ & 70 & $\begin{array}{l}\text { SWP } 55333 \\
\text { LWP } 31135\end{array}$ & $\begin{array}{l}30 \mathrm{~min} \\
18 \mathrm{~min}\end{array}$ \\
\hline N 44:05 & $\begin{array}{lll}52217.8\end{array}$ & -675708.8 & 13.23 & -0.08 & -0.98 & -0.92 & $40 \mathrm{~min}$ & 60 & LWP 31136 & $20 \mathrm{~min}$ \\
\hline N 44:06 & 52137.6 & -675448.0 & 13.38 & -0.12 & -0.98 & -0.90 & $40 \min$ & 50 & $\begin{array}{l}\text { SWP } 55332 \\
\text { LWP } 31134\end{array}$ & $\begin{array}{l}50 \mathrm{~min} \\
30 \mathrm{~min}\end{array}$ \\
\hline N 44:07 & 52207.5 & -675645.3 & 13.39 & -0.10 & -0.99 & -0.92 & $40 \min$ & 40 & - & - \\
\hline N 44:08 & 52147.3 & -675540.3 & 13.44 & -0.07 & -0.96 & -0.91 & $40 \mathrm{~min}$ & 40 & - & - \\
\hline $\mathrm{N} 44: 09^{b}$ & 52226.2 & -675737.8 & 13.71 & -0.08 & -0.94 & -0.88 & $80 \mathrm{~min}$ & 70 & $\begin{array}{l}\text { SWP } 55311 \\
\text { LWP } 31097\end{array}$ & $\begin{array}{r}100 \mathrm{~min} \\
60 \mathrm{~min}\end{array}$ \\
\hline N 44:10 & 52206.3 & -675703.5 & 13.75 & -0.05 & -0.89 & -0.85 & $80 \min$ & 65 & $\begin{array}{l}\text { SWP } 55312 \\
\text { LWP } 31098\end{array}$ & $\begin{array}{l}90 \mathrm{~min} \\
50 \mathrm{~min}\end{array}$ \\
\hline N 44:11 & 52154.8 & -675534.5 & 13.84 & -0.11 & -0.89 & -0.81 & $60 \min$ & 40 & $\begin{array}{l}\text { SWP } 55317 \\
\text { LWP } 31107\end{array}$ & $\begin{array}{l}90 \mathrm{~min} \\
50 \mathrm{~min}\end{array}$ \\
\hline N 44:12 & 52159.5 & -675720.7 & 13.89 & -0.10 & -0.97 & -0.90 & $60 \min$ & 45 & $\begin{array}{l}\text { SWP } 55324 \\
\text { SWP } 55331 \\
\text { LWP } 31122\end{array}$ & $\begin{array}{l}80 \mathrm{~min} \\
70 \mathrm{~min} \\
50 \mathrm{~min}\end{array}$ \\
\hline N 44:13 & $\begin{array}{lll}5 & 22 & 21.6\end{array}$ & -675552.9 & 13.87 & -0.05 & -0.82 & -0.79 & $60 \mathrm{~min}$ & 30 & - & - \\
\hline N 44:15 & $\begin{array}{lll}5 & 2152.4\end{array}$ & -675621.7 & 13.95 & +0.20 & +0.02 & -0.12 & $60 \min$ & 30 & - & - \\
\hline
\end{tabular}

${ }^{a}$ N 44:02 $\equiv$ MACS J0522-679\#027 (MACS = Magellanic Catalogue of Stars,

${ }^{b}$ N 44:09 $\equiv$ MACS J0522-679\#018 Tucholke et al. 1996; see also de Boer et al. 1995).

calibration spectra). Table 5 gives the exposure times and the achieved signal-to-noise ratio.

The spectra have been reduced with MIDAS in a standard way. The wavelength calibration has been performed with He-Ar spectra which have been taken immediately before and after each stellar spectrum. No flux calibration has been performed, instead we normalized the spectra to unity. Figure 5 shows all 14 spectra.

\subsubsection{UV spectra}

Low resolution IUE spectra of 10 of the stars have been observed from 17 th to 20 th July 1995 . We used the large aperture of the IUE. Observing details for these stars are given in Table 5. The spectra are shown in Fig. 6. They have been weakly smoothed with a 5 pixel median filter.

\section{Analysis of the spectra}

\subsection{The optical spectra}

For the classification of the stars with optical spectra we applied different methods: Classification of the spectra by comparison with two atlases of digital spectra, fitting of line profiles to the Balmer lines, and finally estimating the spectral subtypes from the equivalent widths of the He I and some metal lines. From these three methods only the first one worked sufficiently well for all stars. The profile fitting and the equivalent widths can only be regarded as additional constraints on the spectral types and effective temperatures for some of the stars. Since the two latter methods did not add much information, they are not discussed here.

However, the profile fits led to the knowledge of the central wavelengths of the Balmer lines. We could thus derive rough radial velocities for our program stars. They are given in Table 6 . All radial velocities lie in the range from $v_{\mathrm{LSR}}=210 \mathrm{~km} \mathrm{~s}^{-1}(\mathrm{~N} 44: 13)$ to $295 \mathrm{~km} \mathrm{~s}^{-1}$ (N 44:09) with a clear peak of the distribution around $265 \mathrm{~km} \mathrm{~s}^{-1}$. 


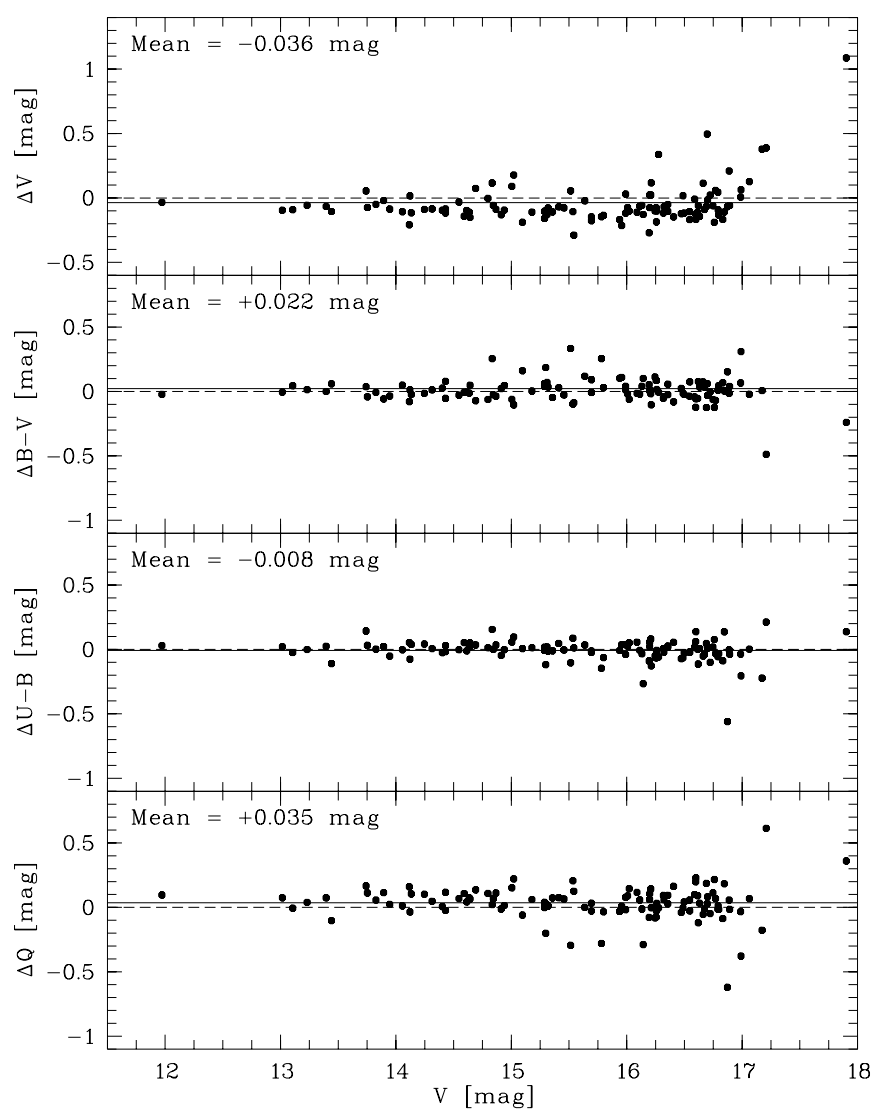

Fig. 3. Comparison of our photometry with that from Lee (1990). Mean values for the differences of $V$ magnitudes, $B-V$ and $U-B$ colours, and for the reddening free parameter $Q$ are given and indicated by the solid line. All differences are in the sense our value minus Lee's value. Zero differences are indicated by dashed lines

We estimate the uncertainty of the radial velocities to $\approx 30 \mathrm{~km} \mathrm{~s}^{-1}$. Our radial velocities are in good agreement with the radial velocities of the molecular lines $\left(v_{\mathrm{LSR}}=\right.$ $283 \mathrm{~km} \mathrm{~s}^{-1}$ ) found by Chin (1995).

The lines in the optical spectra of our stars can be identified by comparison with the atlases of digital spectra of Walborn \& Fitzpatrick (1990) and Fitzpatrick (1991). The atlas of Walborn \& Fitzpatrick (1990) covers O and $B$ type spectra for a large range of spectral subtypes and luminosity classes. The sample spectra are from galactic stars and thus may differ systematically from our target stars due to the lower metallicity of the LMC. The atlas of Fitzpatrick (1991) gives sample spectra from LMC stars and therefore of appropriate metallicity. However, this atlas is only valid for supergiant B stars and thus not useful for all stars of our sample. For the B Ia types in our sample there is a good agreement between the classifications derived from both atlases. Therefore we do also trust the classification from the Walborn \& Fitzpatrick (1990) atlas for the non Ia luminosity class stars.

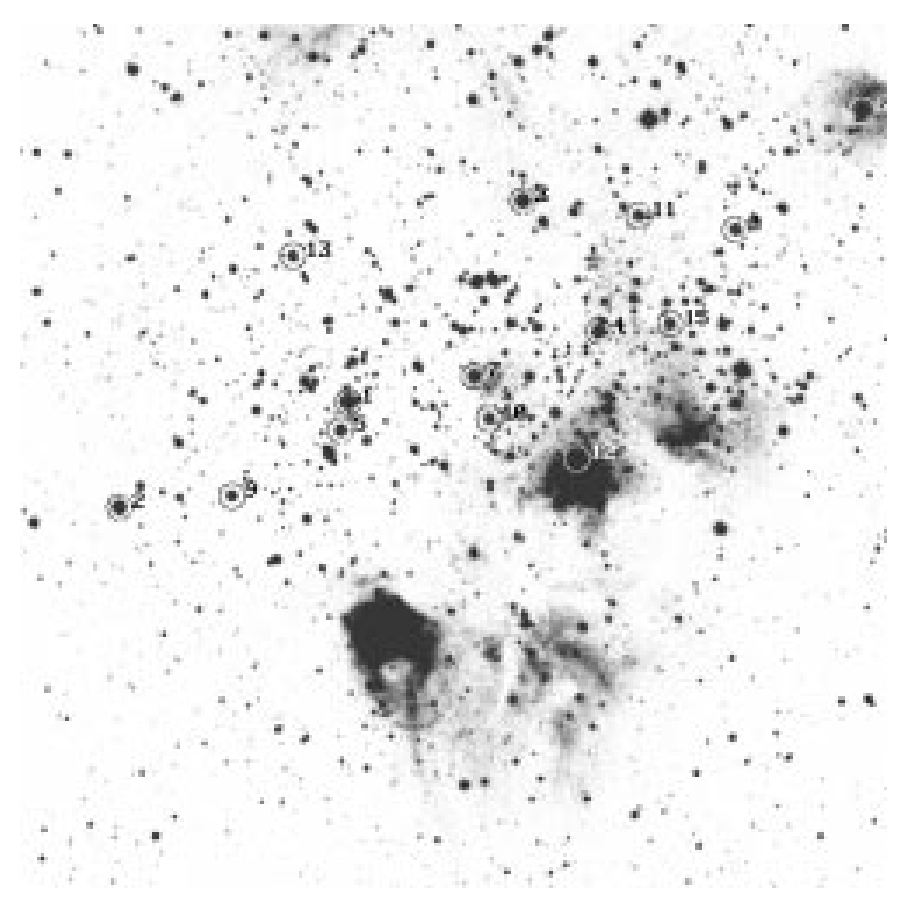

Fig. 4. The target stars for spectroscopy marked on a $V$ exposure of $\mathrm{N} 44$. North is up and east to the left. The size of the field of view is $6.4 \times 6 !^{\prime} 4$

The spectra were taken with the aim to help to refine the age determination of the association. Therefore it is necessary to judge from the spectra if a star is evolved or not, i.e. to determine its luminosity class. However, when comparing the spectra with the sample spectra in the atlases, it soon became clear that a determination of the luminosity class is very difficult. This is mostly due to the fact that for a given spectral type the changes of line widths and line strength ratios with luminosity class are very small.

\subsection{The $U V$ spectra}

From the UV spectra we derived effective temperatures and surface gravities by fitting model atmospheres from the ATLAS 9 program (Kurucz 1992). We used the models with $[M / H]=-0.3$ dex, however, our results do not change when we use $[M / H]=-0.5 \mathrm{dex}$. The photometric magnitudes in the $U, B$, and $V$ passband were converted to fluxes (Heber et al. 1984), plotted together with the IUE spectra and used for the fit of the model atmospheres.

It turned out that there exists no unambiguous solution for $T_{\text {eff }}, \log g$, and $E_{B-V}$ for each spectrum. Therefore we varied $E_{B-V}$ by steps of $0.05 \mathrm{mag}$ in the range suggested by the photometry, thus resulting in several sets of $T_{\text {eff }}, \log g$, and $E_{B-V}$. A constant galactic foreground reddening of $E_{B-V}=0.05 \mathrm{mag}$ was assumed (see e.g. Oestreicher et al. 1995) for which the extinction law of Savage \& Mathis (1979) was used. The remaining portion of the reddening was assumed to be of LMC origin and 


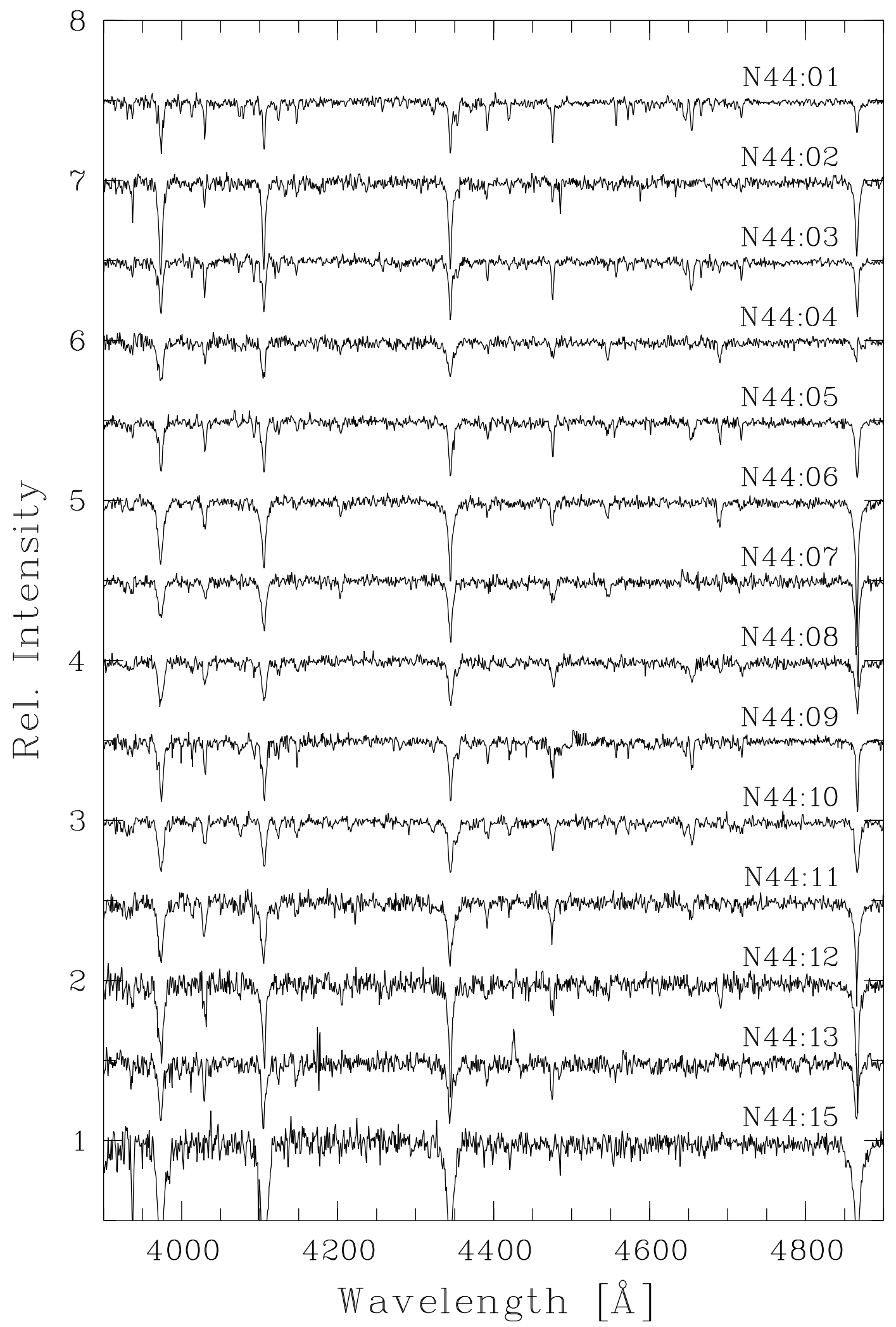

Fig. 5. The normalized optical spectra of the stars in N 44. The wavelength range from 3900 to $4900 \AA$ is shown. There is an offset of 0.5 units in relative intensity from one spectrum to the next 


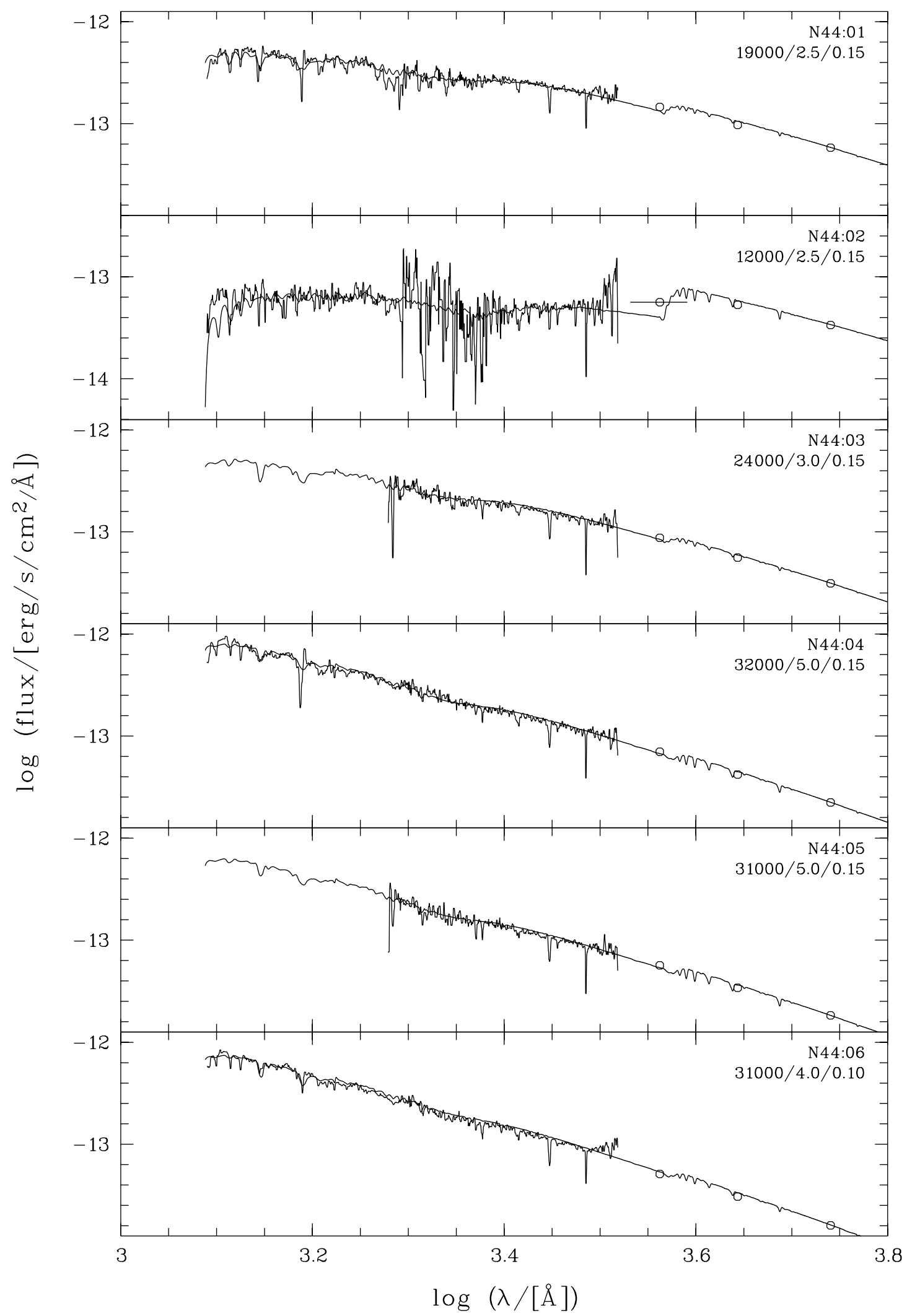

Fig. 6. a) The IUE spectra of the stars 01 to 06 in N 44 and fits of Kurucz (1992) model atmospheres. The open circles denote the magnitudes in $U, B$, and $V$ passbands. The numbers give $T_{\text {eff }}, \log g$, and $E_{B-V}$ of the plotted model. In the case of N $44: 02$ the width of the $U$ filter is indicated by a horizontal line to explain the large deviation between the $U$ magnitude and the flux of the model atmosphere 


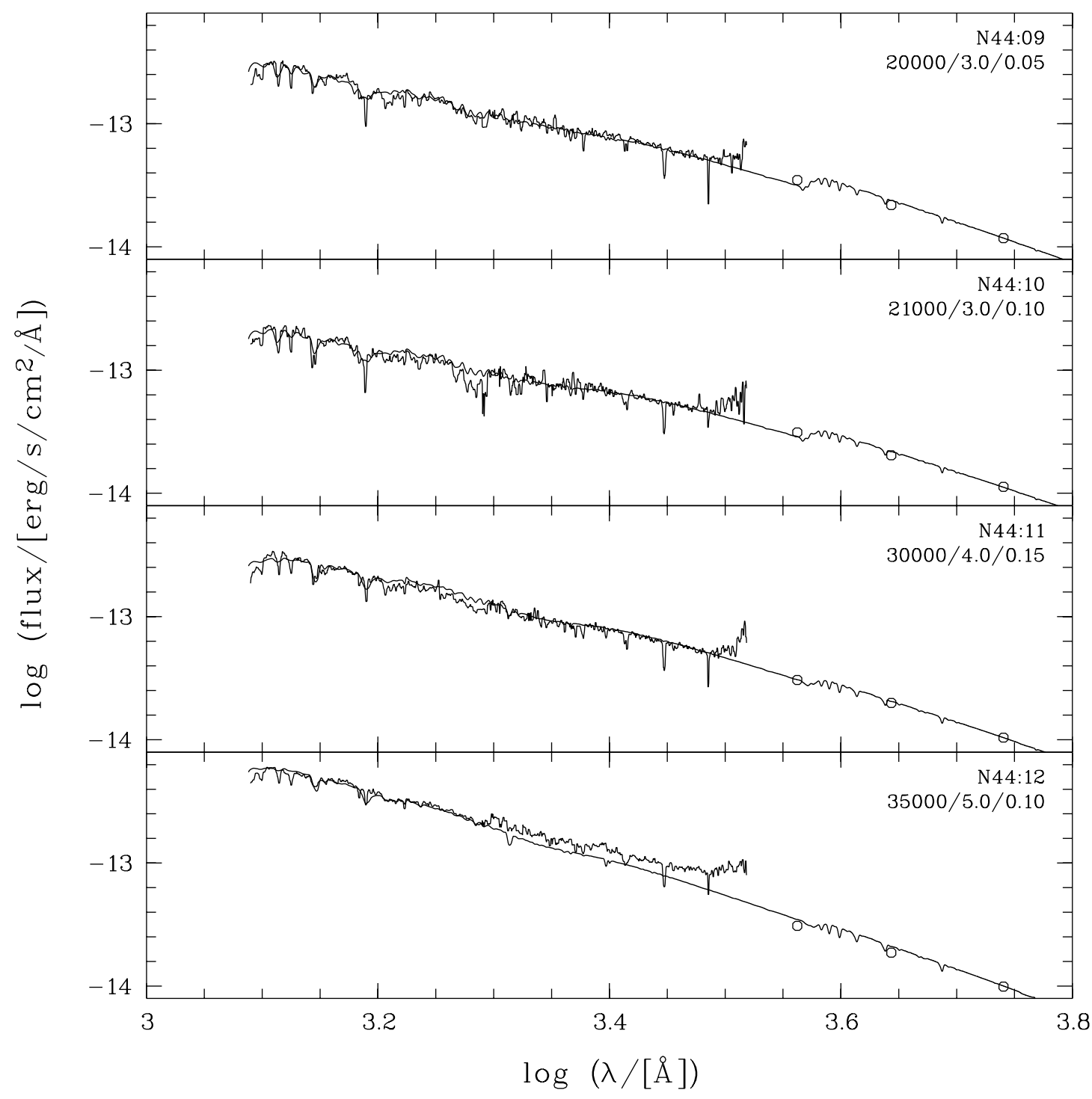

Fig. 6. b) Same as in Fig. 6a for the stars 09 to 12. In the case of N 44:12 the flux of the LWP spectrum seems to be erroneous. This part thus has not been used for the fit of the model atmospheres

the extinction law of Howarth (1983) was used for its correction. For each spectrum one fit is shown in Figs. 6a and $6 \mathrm{~b}$.

In the case of $\mathrm{N}$ 44:03 and $\mathrm{N}$ 44:05 no SWP spectrum was available, thus the fit of the model atmospheres had to rely on the LWP spectrum and the optical flux values alone. This makes those fits more uncertain. In the case of N 44:12 we found a problem with the flux in the LWP spectrum. It seems that LWP and SWP do not fit properly together, although the exposure times were corrected accordingly for both spectra before combining them. There is too much flux in the LWP compared to both, the SWP spectrum and the photometry. A model atmosphere which fits to both parts of the spectrum is in principle possible, but it would totally fail to reproduce the flux values from the photometry.

\subsection{Final classification of the stars with spectra}

We then collected all information from the methods described above to determine the final classification for our sample stars. Due to the difficulties in deriving a reliable luminosity class from the comparison with the spectral atlases, we used the photometric information to derive the luminosity classes by comparison with the tables of Schmidt-Kaler (1982), whereas we took the spectral subtypes from our classification. This led to our final classifications which are summarized in Table 6. For each spectral type we give the corresponding effective temperature as tabulated by Schmidt-Kaler (1982).

In Table 6 we do also give the spectral types which were derived by Oey \& Massey (1995). These are mostly in fair agreement with our classification. The largest 
Table 6. Spectral types, effective temperatures and radial velocities of our sample stars and comparison of identifications, spectral types and effective temperatures with Oey \& Massey (1995)

\begin{tabular}{llccllc}
\hline & & & & \multicolumn{3}{c}{ Oey \& Massey $(1995)$} \\
\cline { 5 - 7 } Star Id. & Spectral type & $T_{\text {eff }}[\mathrm{K}]$ & $v_{\text {rad,LSR }}\left[\mathrm{km} \mathrm{s}^{-1}\right]$ & Id. & Spectral type & $T_{\text {eff }}[\mathrm{K}]$ \\
\hline N 44:01 & B1 Ia & 20800 & 265 & lh47-373A & B1 Ib & 20300 \\
N 44:02 & B8/9 Iab & 10700 & 250 & - & - & - \\
N 44:03 & B0.5 Iab & 24000 & 265 & lh47-237 & B0.5 I & 23100 \\
N 44:04 & O7 III & 37000 & 220 & $l h 47-154$ & O7 V((f)) & 40100 \\
N 44:05 & O9 III & 32000 & 270 & $l h 47-381$ & O8.5 III(f) & 35000 \\
N 44:06 & O8 III & 34700 & 255 & - & - & - \\
N 44:07 & O9 III & 32000 & 265 & $l h 47-280$ & O7 III(f) & 38100 \\
N 44:08 & B0 III & 29000 & 290 & $l h 47-47$ & O9.5 V & 35000 \\
N 44:09 & B1 III & 24000 & 295 & $l h 47-444$ & B0.5 I & 23100 \\
N 44:10 & B1 III & 24000 & 270 & $l h 47-268$ & B1 Ib & 20300 \\
N 44:11 & B0.2 III & 28000 & 230 & $l h 47-110$ & B0.5 V & 25400 \\
N 44:12 & O8 V & 35800 & 260 & $l h 47-186$ & O7.5 V + ne & 39100 \\
N 44:13 & B1.5 II & 20000 & 210 & $l h 47-419$ & B1.5 III & 20000 \\
N 44:15 & A0 Ib & 9730 & 250 & - & - & - \\
\hline
\end{tabular}

deviation occurs for $\mathrm{N}$ 44:07 with a discrepancy of two spectral subtypes and for $\mathrm{N}$ 44:08 with a large difference in temperature. We found luminosity class III for the stars $\mathrm{N}$ 44:04 to $\mathrm{N} 44: 10$, which is mainly due to the comparison of the absolute magnitudes with the tables of SchmidtKaler (1982), whereas Oey \& Massey (1995) attribute quite different luminosity classes to those stars.

\section{Determination of the age}

We will first derive the age from our CMD by comparison with isochrones. This result will then be compared with the distribution of stars in the theoretical plane, i.e. in the Hertzsprung-Russell diagram (HRD).

\subsection{From photometry alone}

The age of the association has been derived by fitting isochrones to the CMD which were calculated from the stellar evolutionary models of Schaerer et al. (1993). These models include moderate convective core overshooting and new opacities (Rogers \& Iglesias 1992). We used the tracks for a metal content of $Z=0.008$, which is appropriate for most young LMC populations (see e.g. Spite et al. 1993; Hill et al. 1995). For the isochrone fit we used a LMC distance modulus of $18.5 \mathrm{mag}$ (Westerlund 1990).

Using both CMDs the best fit to the youngest population is reached for an isochrone with an age of $5 \mathrm{Myr}(\log ($ age $/[\mathrm{yr}])=6.7)$ and a reddening of $E_{B-V}=$ $0.22 \mathrm{mag}$. In the $V$ vs. $B-V$ diagram also an age of 6 Myr would give a good fit. The foreground reddening in this region of the LMC is very low: from the map of Oestreicher et al. (1995) we find 0.04-0.06 mag, the map of Schwering \& Israel (1991) gives $0.06-0.08$ mag. Thus the major part of the reddening is due to LMC gas. Due to the huge gas amount in the region (cf. Fig. 1) this is not astonishing and we have to expect a strongly varying reddening within our field of view. Oey \& Massey (1995) give a lower mean reddening of $E_{B-V}=0.11$ mag.

The CMD with the 5 Myr isochrone is shown in Fig. 7. In the $M_{V}$ vs. $(B-V)_{0}$ plane the fit of the isochrone is quite good. In the $M_{V}$ vs. $(U-B)_{0}$ plane the fit near the turnoff region of the main sequence is bad. The shape of the upper main sequence is strange and can not be fitted with any isochrone. There is also an unusual high scatter in the $U-B$ colour. A wrong $U$ calibration does not seem to be very probable, because first this would not produce scatter but a common shift of all stars, and second the agreement with the $U-B$ colour of Lee (1990, Fig. 3) is good.

\subsection{From photometry and spectroscopy}

The stars for which a spectral type was derived could easily be placed in the HRD. For all other stars we used the transformation equations given by Massey et al. (1995b) to derive $M_{\text {bol }}$ and $T_{\text {eff }}$. In these equations the reddening for stars with $Q<-0.4 \mathrm{mag}$ is derived from the photometry individually for each star. Figure 8 shows the distribution of the derived reddening values. The distribution shows a clear peak near $E_{B-V}=0.22 \mathrm{mag}$, thus confirming our fit in the observational plane. There are some stars which show unusual high reddenings. These are artefacts of an uncertain $U$ magnitude of faint red stars.

Figure 9 shows a map of the reddening on the position of N 44. Due to the above mentioned uncertain high reddening values, only stars with $E_{B-V}<0.8$ mag have 


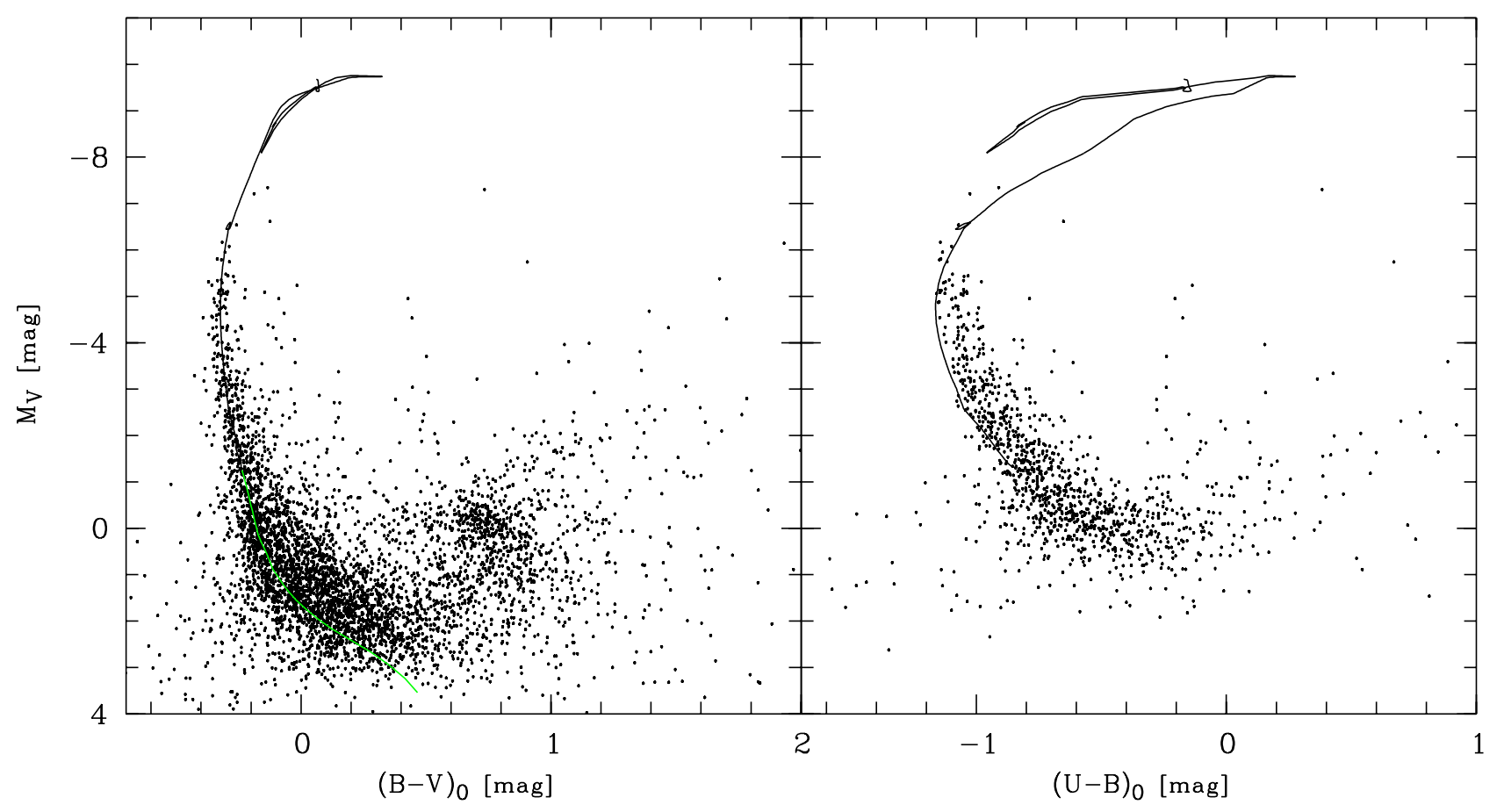

Fig. 7. CMDs for all stars in the field of view, $M_{V}$ vs. $(B-V)_{0}$ (left panel) and $M_{V}$ vs. $(U-B)_{0}$ (right panel), with an overplotted isochrone. The isochrone is calculated from the modes of Schaerer et al. (1993) for an age of 5 Myr and a metallicity of $Z=0.008$. A distance modulus of $18.5 \mathrm{mag}$ and a reddening of $E_{B-V}=0.22 \mathrm{mag}$ have been assumed

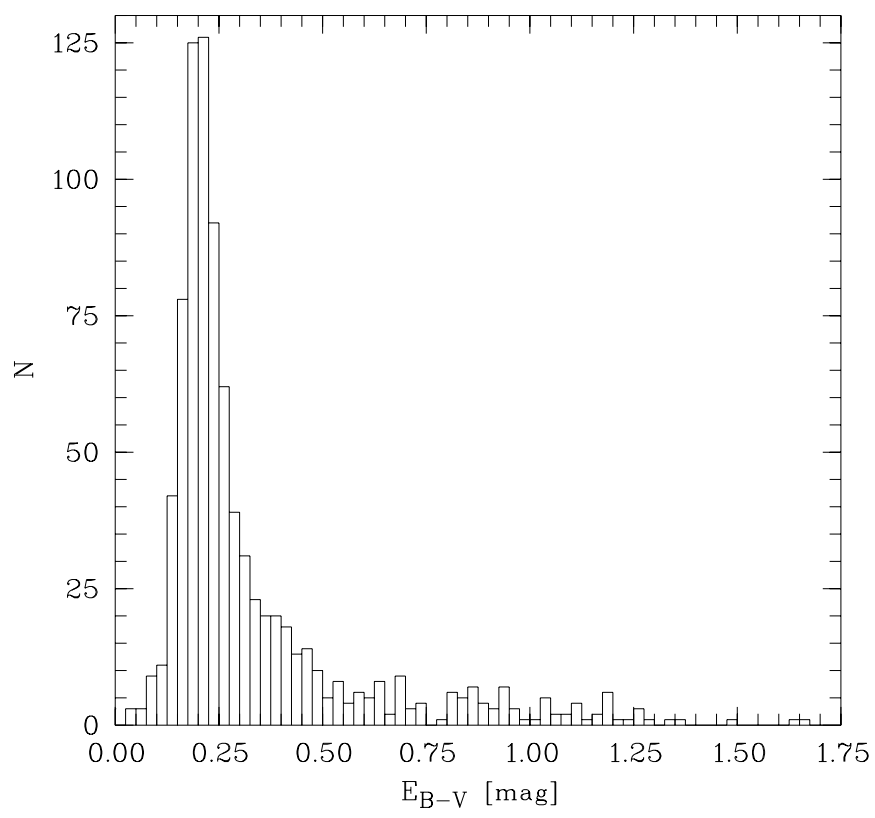

Fig. 8. Distribution of the reddening values derived from conversion of the photometry to $T_{\text {eff }}$ and $\log L$. The distribution peaks near $E_{B-V}=0.22 \mathrm{mag}$, which is the reddening derived from the isochrone fit been used to construct this map. Also only stars with $Q<-0.4$ mag have been used because only for those stars an individual reddening was derived from the photometry. The map shows that the reddening in the inner part of the shell is not very strong and near the mean value of 0.22 mag. South-west of the shell, between N 44B and $\mathrm{N} 44 \mathrm{C}$, we find a peak of the reddening which could originate from the higher gas and dust content of this region (see Fig. 1).

Figure 10 shows the HRD with overlayed tracks (left panel) and isochrones (right panel) from the models of Schaerer et al. (1993). The stars hotter than the main sequence band are faint stars with uncertain, i.e. too blue colours. Along the entire main sequence we recognize the same effect that we have already seen in Fig. 7 . There is no single isochrone which gives a good fit, instead the stars spread over the entire main sequence band. This spread has the same reason than the scatter in the $V$ vs. $U-B$ CMD (Fig. 7, right panel), i.e. an unusual high scatter of the bright stars in $U-B$, which is brought into the HRD via the transformation equations. We do not understand this high scatter, but it is also present in the HRD of Oey \& Massey (1995, their Fig. 4) and thus may be an intrinsic feature of LH 47. 


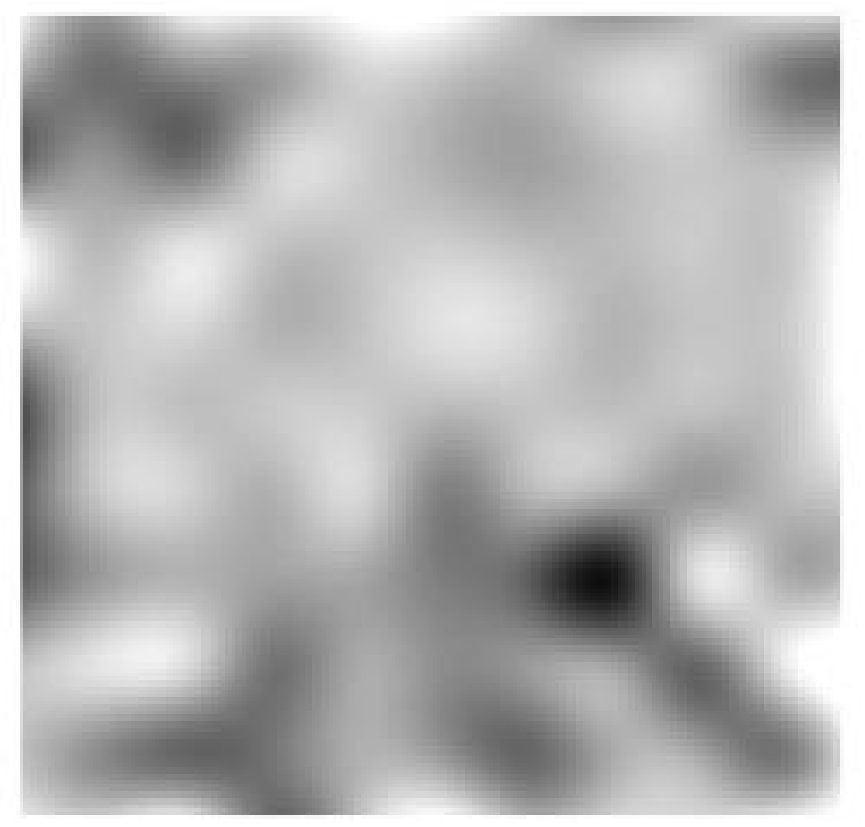

0.1 0.4

Fig. 9. Map of the reddening derived from conversion of the photometry to $T_{\text {eff }}$ and $\log L$. Light grey values mean slight reddening, dark grey values denote strong reddening. The field is approximately the same as in Fig. 4. Only stars with $E_{B-V}<0.8 \mathrm{mag}$ and $Q<-0.4 \mathrm{mag}$ have been used for this map

\subsection{Discussion}

From the CMDs we find an age of 5 to 6 Myr for LH 47 in N 44. Oey \& Massey (1995) find 10 Myr for LH 47 (inside the central shell of N 44), Kontizas et al. (1996) give an upper limit of $3 \mathrm{Myr}$ for the associations LH 47, 48 and 49.

Oey \& Massey (1995) find a significant difference in the ages of the stars inside (10 Myr) and outside (5 Myr) the shell. We do not see such a difference. From Fig. 4 it is clear that there are some bright stars in the very centre of the shell. Those stars include N 44:01 and N 44:05, for which we do have spectra. Our spectral classification of those stars is in good agreement with that of Oey \& Massey (1995). These two stars are as young as $5 \mathrm{Myr}$, thus implying that there is no age difference between the interior and exterior of the shell. However, they could still be located at the border or outside of the shell, so that they are only projected in front of the centre. In Fig. 11 we compare the CMDs of the stars inside and outside the shell. Inside means all stars nearer than 1.9 to the center and outside means all stars more far than 2.5 from the center of the shell. In this way we selected only stars which are really inside or outside the gas ring of $\mathrm{N} 44$, omitting stars which fall on the gas ring. The $5 \mathrm{Myr}$ isochrone is also shown for easier comparison of both plots. As we concluded already from the HRD, we do not see a difference of the ages of the stellar population inside and outside the shell.

Due to the calibration problem in the work of Oey \& Massey (1995; see Sect. 2.1), the age difference found by those authors may be an artefact of the photometric offset between their CCD fields. This seems very plausible because of the fact that a distinction between inside and outside the shell in their photometry may be traced back to a different calibration of their CCD fields which had only a narrow overlap.

The very hot star observed by Stasińska et al. (1986, their \#2) is also included in our photometry. For this star we find $M_{V}=-5.06 \mathrm{mag},(B-V)_{0}=-0.31 \mathrm{mag}$ and $(U-B)_{0}=-1.16 \mathrm{mag}$. After transformation to the theoretical plane, this leads to a temperature of $38500 \mathrm{~K}$ (i.e. $\left.\log T_{\text {eff }}=4.586\right)$ and $\log \left(L / L_{\odot}\right)=5.411$. Its reddening is $E_{B-V}=0.226 \mathrm{mag}$ and thus only slightly higher than the average reddening. The star lies well in the main sequence band, confirming the classification as a normal $\mathrm{O}$ star by Oey \& Massey (1995; their lh47-338) and Pakull \& Motch (1989) but not confirming the very high temperature given by Stasińska et al. (1986).

One of our goals of the spectroscopy and the transformation of the photometry to $T_{\text {eff }}$ and $\log L$ was to refine the age determination from the pure $B V$ photometry. This was not as successful as we expected due to the not understood scatter in the $U-B$ colour, which is transformed also into the HRD. The inspection of Fig. 10 however shows that the stars with spectra suggest a minimum age of the association of 4 or $5 \mathrm{Myr}$, thus confirming our result from the photometry.

\section{Luminosity function}

The luminosity function is a good tool to compare the stellar content of different regions. It gives a first hint on the ratio between massive (bright) and less massive (faint) stars. However, different evolutionary states of the stars smear out some properties. But in contrast to the mass function no stellar evolutionary models or other theories are needed for the construction of the luminosity function. We assume that the luminosity function is a power law with

$\log N\left(M_{V}\right) \propto \gamma M_{V}$

where $\gamma$ is the slope of the luminosity function.

The luminosity function has been constructed from main sequence stars. Only stars with $M_{V}>-6.5 \mathrm{mag}$ and stars which do not belong to the "red clump" have been selected; in total these are 3522 objects. The star counts have been binned to interval widths of $1 \mathrm{mag}$. Figure $12 \mathrm{a}$ shows the luminosity function found for all those stars. The error bars represent $\sqrt{N}$ errors. In the range from $M_{V}=-6$ to +2 mag the assumption of a power law seems 


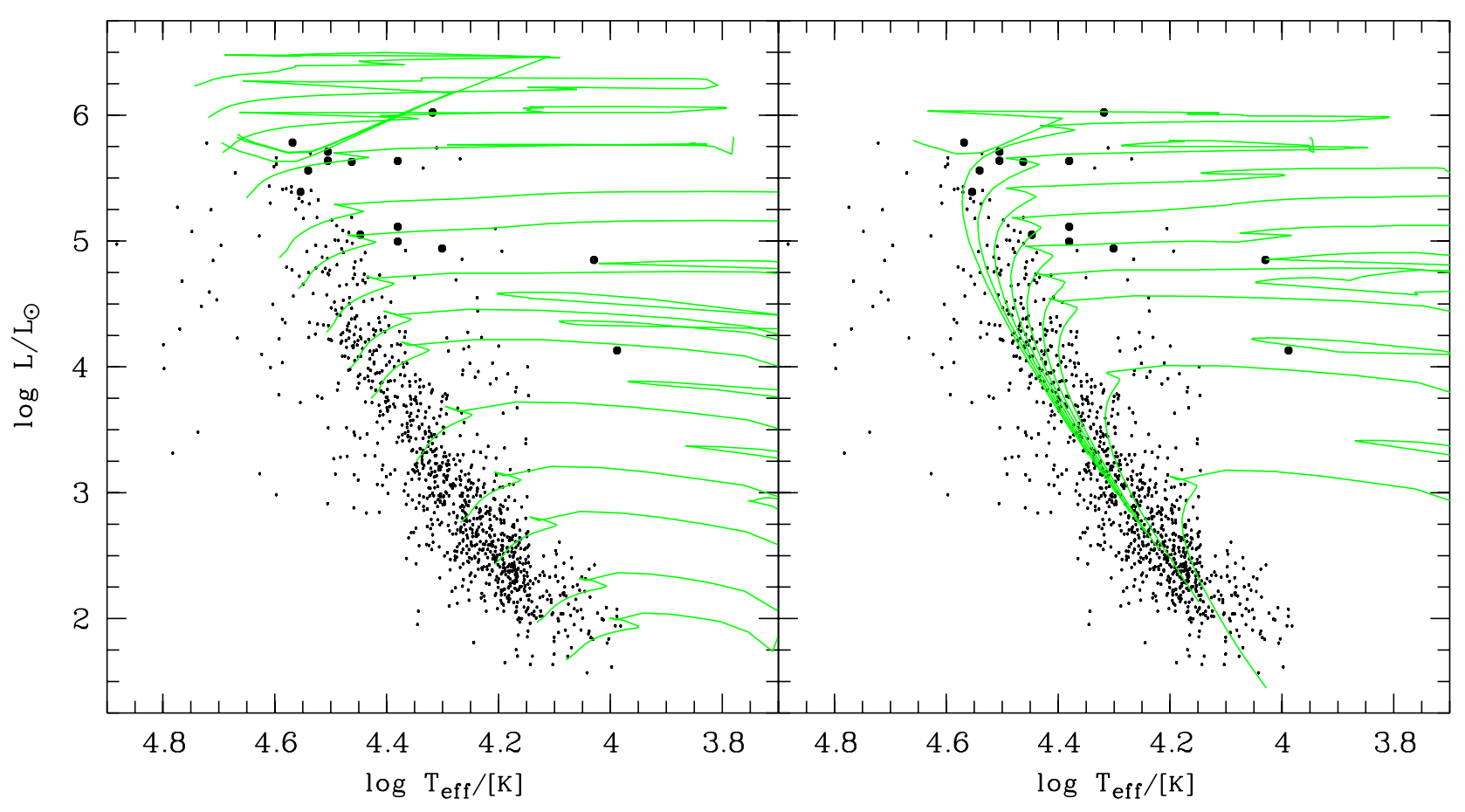

Fig. 10. HRD of the stars in N 44. The stars for which spectra exist are marked by the larger symbols. On the left panel tracks with $Z=0.008$ from Schaerer et al. (1993) for masses from 120 down to $2.5 \mathcal{M}_{\odot}$ have been overplotted (masses $=120,85,60$, $40,25,20,15,12,10,7,5,4,3$, and $\left.2.5 \mathcal{M}_{\odot}\right)$. On the right panel isochrones for ages of 4 Myr to $100 \mathrm{Myr}(\log$ age $=6.6,6.7$, $6.8,6.9,7.0,7.1,7.2,7.5$, and 8.0) calculated from those tracks have been overplotted

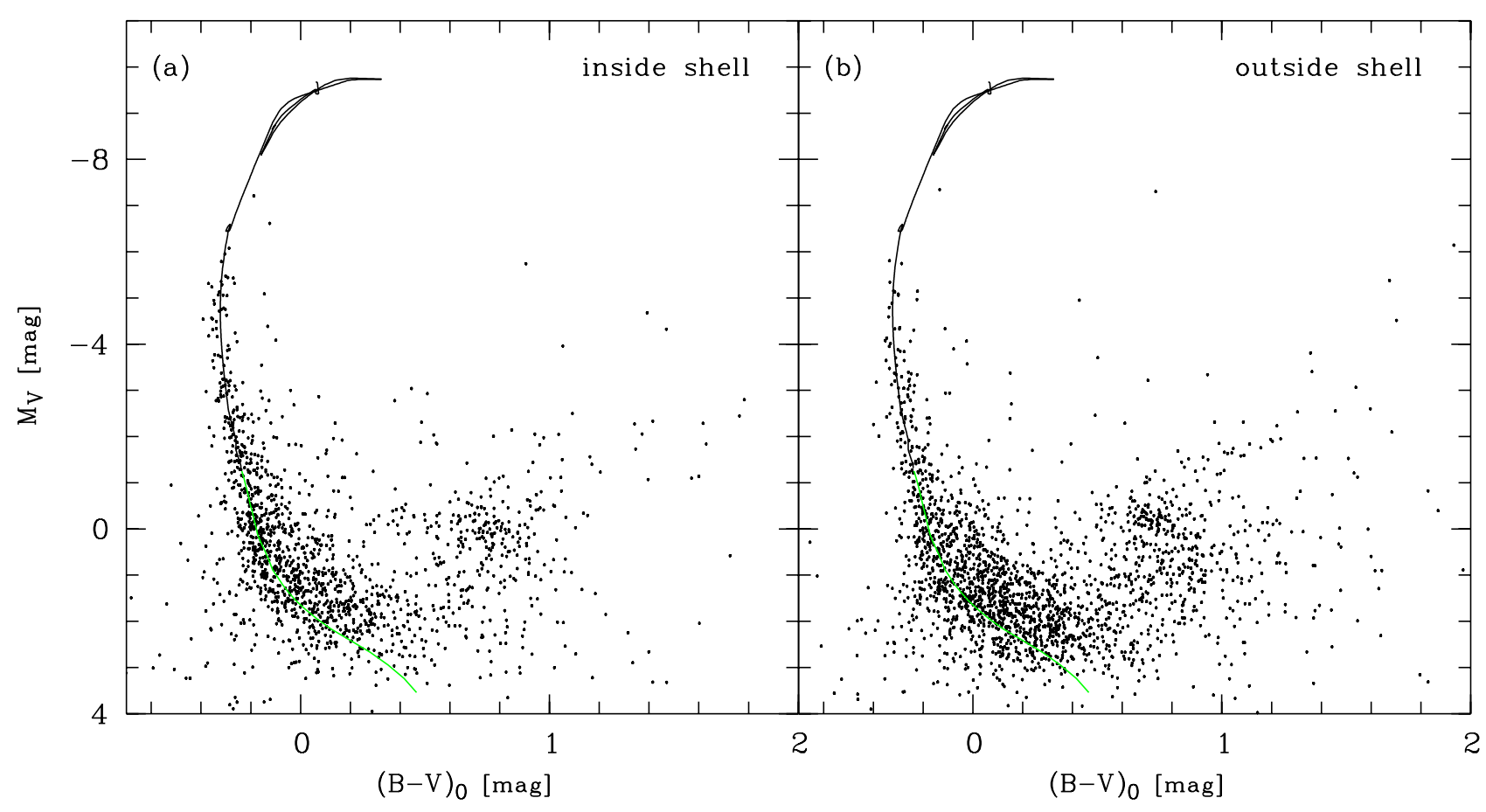

Fig. 11. Comparison of the CMDs of stars a) inside and b) outside the shell of N 44. An isochrone with an age of 5 Myr is overlayed. A reddening of $E_{B-V}=0.22 \mathrm{mag}$ has been assumed for both plots 


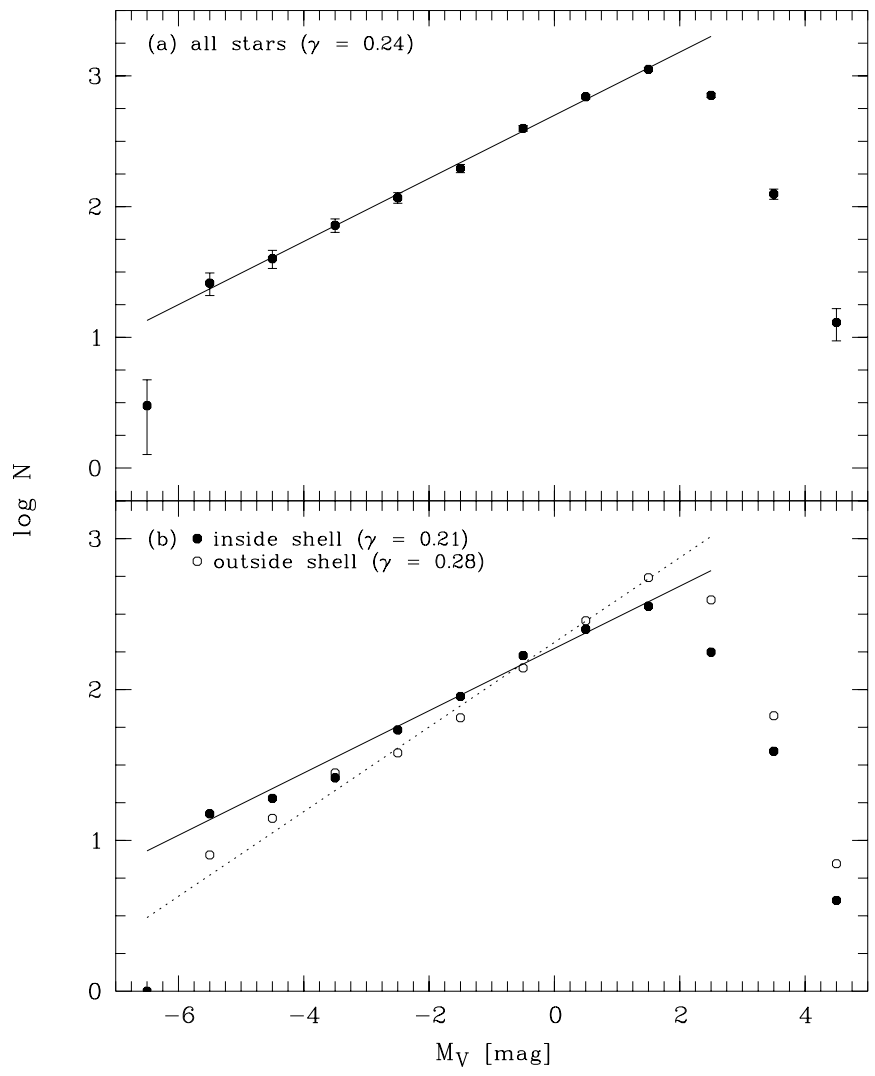

Fig. 12. Luminosity function (LF) for LH 47/48. a) The LF for all 3522 main sequence stars. b) Comparison of the LF of inside the shell N 44 (filled circles) and outside (open circles). The slopes of weighted linear regressions are given (inside: straight line, outside: dotted line)

to be correct. The first bin $\left(M_{V}=-6.5 \mathrm{mag}\right)$ contains too few stars, probably some stars are already missing due to evolutionary effects. At $M_{V}=+2 \mathrm{mag}$ (i.e. $V \approx 21 \mathrm{mag}$ ) incompleteness of the data sets in. The straight line represents a weighted linear regression for the range $M_{V}=-6$ to $+2 \mathrm{mag}$ (i.e. $V=13$ to $21 \mathrm{mag}$ ). The slope of the line is $\gamma=0.24 \pm 0.02$.

Figure $12 \mathrm{~b}$ shows a comparison of the luminosity function between the interior and the exterior of the superbubble N 44. For the inner part all main sequence stars with a distance of less than 1'.9 from the centre of the shell have been taken into account (1204 stars), for the outer part only stars more distant than 2.5 (1601 stars) from the centre have been selected. Error bars have been omitted in Fig. 12b for clarity. There is a clear difference of the luminosity function between the two samples. In the inner part the luminosity function flattens significantly towards brighter stars. An error-weighted linear regression gives $\gamma=0.21 \pm 0.02$ for the interior of the shell and $\gamma=0.28 \pm 0.02$ for the exterior. The fits have been calculated for the same magnitude range as in Fig. 12a, i.e. from $M_{V}=-6$ to $+2 \mathrm{mag}$. The solid line represents the fit for the inner region, the dotted line the fit for the outer region.

The slope of the luminosity function for the entire region $(\gamma=0.24)$ is in good agreement with the value of $\gamma=0.26$ found for the luminosity function of NGC 196265-66-70 (LH 58; Will et al. 1995b). Malumuth \& Heap (1994) found $\gamma=0.26$ for the inner region of 30 Dor and $\gamma=0.33$ for the outer parts ${ }^{1}$. For NGC 595 in M 33 Malumuth et al. (1996) give $\gamma=0.28$. For more slopes of luminosity functions in recent papers see Will et al. (1995b).

\section{Mass function}

We have two possibilities to derive the slope of the IMF. First, we can use the isochrone which was found to fit best to the CMD to convert the apparent magnitudes of the main sequence stars to masses. This method has been used in our recent papers (Will et al. 1995a,b; Vázquez et al. 1995) and proved to be very successful. Since we are dealing with hot stars and we do have observations in the $U$ passband, we can also use the transformation equations of Massey et al. (1995b) to transform our CMD to a HRD. This enables us to overplot tracks from stellar evolutionary models and to count stars in certain mass intervals. Both methods will be used here and their results will be compared.

We did not apply any completeness correction (similar to e.g. Oey \& Massey (1995) but in contrast to e.g. Will et al. 1995a,b). Our photometry of N 44 is so deep (see Figs. 2 and 7 ) that we expect a high contamination of LMC field stars in the faint star regime. But, since we do not have any observations of a comparison field to perform a statistical field star subtraction, the faint star regime would, even with a completeness correction, be useless for us. The luminosity function (Fig. 12) suggests that our photometry is complete down to $M_{V} \approx 1.5$ mag (i.e. $V \approx 20.5 \mathrm{mag}$ ), corresponding to $\mathcal{M}=2.3 \mathcal{M}_{\odot}$ or $\log \mathcal{M}=0.36$.

\subsection{Results}

\subsubsection{From $B V$ photometry}

The $5 \mathrm{Myr}$ isochrone was used to derive a relation between the absolute magnitude $M_{V}$ and the mass of all stars on the main sequence (same criteria as for the luminosity function). The stars were binned in intervals of 0.1 in log mass. We assume a power law form of the IMF for the restricted mass range under investigation and use the equation

$\mathrm{d} N \propto \mathcal{M}^{-x} \mathrm{~d} \log \mathcal{M}$

$\overline{1}$ Malumuth \& Heap (1994) and Malumuth et al. (1996) use $B$ magnitudes and the equation $\log \Phi\left(L_{B}\right) \propto-0.4 \alpha M_{B}$, where $-0.4 \alpha=\gamma$. 
where $x$ is the slope of the mass function. In this notation the value for the slope of the IMF in the solar vicinity is $x=1.35$ (Salpeter 1955).

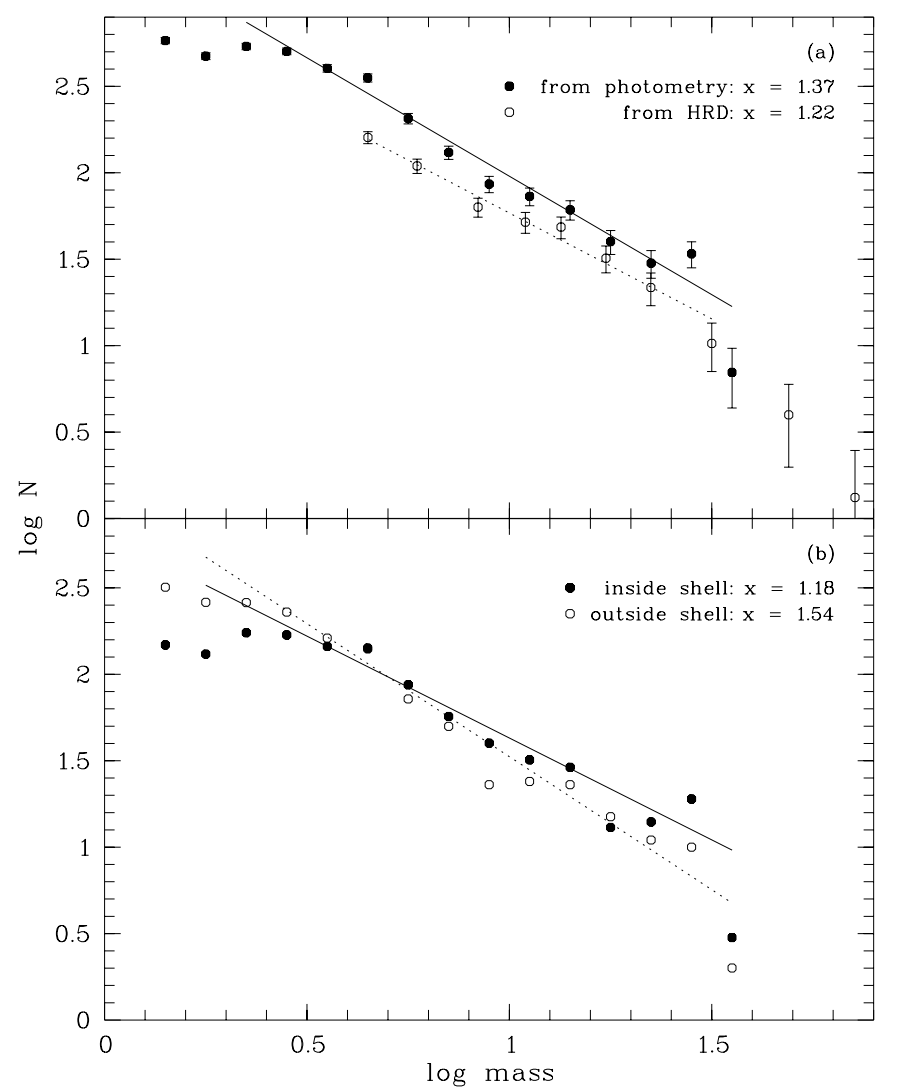

Fig. 13. Initial mass function (IMF) for $\mathrm{LH} 47 / 48$. a) Comparison of the IMF as derived from $B V$ photometry (filled symbols, solid line) and from the HRD (open symbols, dotted line). b) Comparison of the IMF of inside (filled circles, solid line) and outside (open circles, dotted line) the shell of N 44. In both plots the slopes of weighted linear regressions are given

Figure 13 shows the result. In Fig. 13a the star counts for the entire field of view are plotted vs. the logarithm of the mass. $\sqrt{N}$ error bars are also plotted. In the mass range from $2.5 \mathcal{M}_{\odot}(\log \mathcal{M}=0.4)$ to $30 \mathcal{M}_{\odot}(\log \mathcal{M}=$ 1.5) the assumption of a power law seems reasonable. The lower limit fits well with the incompleteness limit derived from the luminosity function. A weighted linear regression in the mentioned mass range gives a slope of the mass function of $x=1.37 \pm 0.10$. The solid line in Fig. 13a shows this fit.

The luminosity function implied a difference between the stellar populations inside and outside of the superbubble N 44A (see Fig. 12b). We used the same selection criteria as for the luminosity function (inside: all stars in a circle with a radius of $1 ! 9$; outside: all stars outside a circle of radius 2.5 ) to investigate this for the IMF. Figure $13 \mathrm{~b}$ shows the star counts for both regions. The filled sym- bols show the mass function for the interior of the shell. A weighted linear regression for the same mass range as mentioned above gives a slope of $x=1.18 \pm 0.15$ (solid line). The open circles give the result for the exterior region where we find a slope of the IMF of $x=1.54 \pm 0.15$ (dotted line).

\subsubsection{From the HRD}

We used a plot similar to the one shown in Fig. 10 (left panel) to count the stars between the stellar evolutionary tracks on the main sequence band of the HRD. The blue stars leftwards of the main sequence are scattered into this region due to uncertain photometry and thus have been omitted for the counting. The red stars to the left of the main sequence near $10 \mathcal{M}_{\odot}$ have been excluded. However, since we want to compare our results with those obtained by Oey \& Massey (1995), we did count all evolved stars which are more massive than $12 \mathcal{M}_{\odot}$.

The star counts between the tracks have been normalized to the same bin size ( 0.1 in log mass) than those from the photometry and have been plotted in Fig. 13a (open circles). The error bars are $\sqrt{N}$ errors. The IMF derived in this way lies clearly below the one from the photometry. This is a direct influence of the fact that for a placement in the HRD a star has to be detected in all three passbands $U, B$ and $V$. In the $U$ passband our observations are not very deep and incomplete already on the upper main sequence. However, the slope of $x=1.22 \pm 0.10$ derived for the mass range below the turn off point of the main sequence is quite similar to that from the $B V$ photometry. We recognize a clear upturn of the IMF slope for stars more massive than $20 \mathcal{M}_{\odot}(0.1$ in $\log \mathcal{M})$. For the mass range from 12 to $85 \mathcal{M}_{\odot}$ we find a slope of $x=2.1$.

\subsection{Discussion}

We found very similar slopes for the IMF from both methods described above. This is somewhat surprising because of the lower number of stars in the HRD compared to the $B V$ CMD. Using the HRD offers the possibility to count stars up to much higher masses than the turn off point of the best fitting isochrone. This leads to a steeper mass function slope since some stars may have already vanished and are thus not counted, although they were created together with the stellar population which is still observed. The problem has already been discussed by Will et al. (1995b) for the LMC OB association LH 58. Values derived by this method should therefore be treated with care.

Oey \& Massey (1995) give an IMF slope of $x=$ $1.3 \pm 0.2(\Gamma=-1.3$ in their notation $)$ for the association. This value was derived by the second method described above, i.e. counting the stars between the tracks in the HRD. It fits well to the results derived from both methods in this paper. Oey \& Massey (1995) do not find 
a significant difference between the IMF slopes inside and outside the shell. We find a change of $x$ from 1.2 inside to 1.5 outside the shell, i.e. we find relatively less massive stars outside the shell than inside where the association LH 47 is located. This is the opposite direction of IMF slope difference reported by Parker et al. (1992) for LH 9 and LH 10, the associations inside and on the rim of the superbubble N 11. There more massive stars seem to have formed in the association which is located on the rim of the superbubble.

The overall IMF slope found for LH 47 fits well within recent derived IMF slopes in the LMC as well as in our galaxy. Will et al. (1995b) found $x=1.2$ for LH 58 in the LMC. For that association Garmany et al. (1994) found $x=1.6$, which was updated to $x=1.4$ in Massey et al. (1995b). The later paper also compiled other IMF studies of OB associations in the Magellanic Clouds and of open clusters and associations in our galaxy (their Table 5). The Magellanic Cloud associations show IMF slopes in the range from $x=1.1$ to 1.6. In the case of the galactic clusters/associations the range is larger, from $x=0.9$ up to $x=2.1$. Other IMF studies of globular clusters and galactic clusters have been discussed in Will et al. (1995a,b) and Hill et al. (1994).

There is a clear tendency to find IMF slopes near the Salpeter (1955) value. This strongly supports the idea of an universal shape of the IMF. We think that the differences found between the stellar aggregates are more due to scatter (especially at the massive end of the IMF), quality of the observations (i.e. completeness), and the analyzing methods (i.e. counting of stars in CMD or HRD) than due to real differences of the IMF of the objects under investigation. For a comparison of studies which analyze different mass ranges one should also keep in mind that the representation of the IMF with a power law is only valid for a narrow mass range. For larger ranges, i.e. for mass ranges of more than one order of magnitude, the Miller \& Scalo (1979) law should be applied.

\section{Summary and conclusions}

We have analyzed photometric and spectroscopic data of the association LH 47 in the superbubble N 44. The photometry has been reduced in a standard way. The optical and UV spectra were used together with the photometric information to derive spectral classifications and temperatures for those stars. For all other stars the $U B V$ photometry was transformed to the theoretical plane with the conversions given by Massey et al. (1995b). We found a large scatter of the bright stars along the main sequence in the $U-B$ colour. This scatter, which is also present in the study of Oey \& Massey (1995), leads to a broadening of the main sequence in the HRD and thus to an unclear age determination from the HRD.

From an isochrone fit to the CMD we find an age for the youngest population of 5 to $6 \mathrm{Myr}$ and a mean redden- ing of $E_{B-V}=0.22 \mathrm{mag}$. We do not find strong evidence for an age difference between the interior and the exterior of the shell. However, this conclusion is based mainly on two young stars seen inside the shell. They could also be located at the border or outside of the shell and only being projected in front of the centre. Due to the scatter in the $U-B$ colour, which is transfered to the HRD, the HRD did not help to improve the age determination from the photometry. However, the HRD and the spectroscopically investigated stars suggest a lower age limit for the association of $4-5 \mathrm{Myr}$, in perfect agreement with the age determination from the $B V$ photometry.

From the transformation of our $U B V$ photometry to $T_{\text {eff }}$ and $\log L$ we get to know an individual reddening for all stars with $Q<-0.4$ mag. The distribution of these reddening values peaks at $E_{B-V}=0.22 \mathrm{mag}$, thus confirming the mean reddening derived from the isochrone fit to the $B V$ CMD. From the reddening values we construct a reddening chart of $\mathrm{N} 44$. In the centre of the shell we find reddening values around the mean value. South-west of the central shell, between N 44B and N 44C, we find a peak of the reddening which may be associated with the higher gas content in this region.

Chin (1995) found the peak of the ${ }^{13} \mathrm{CO}$ emission very concentrated compared to the $\mathrm{H} \alpha$ and $\mathrm{H}$ I emission and the CO map of Cohen et al. (1988). Using our grid of astrometric reference stars, we find that the position of the ${ }^{13} \mathrm{CO}$ emission given by Chin (1995) lies exactly in the centre of the central shell of N 44 . The N 44 superbubble with LH 47 inside is therefore at the front side of a molecular cloud. It appears probable, that the expansion of $\mathrm{N} 44$ will trigger more star formation in that molecular cloud.

The molecular transitions studied by Chin (1995) showed radial velocities of $v_{\mathrm{LSR}} \approx 280 \mathrm{~km} \mathrm{~s}^{-1}$, which is in good agreement with the radial velocities we found for our spectroscopically investigated stars.

From the main sequence stars in the CMD we construct the luminosity function. We find a slope of $\gamma=$ $0.24 \pm 0.02$ for a power law representation of the luminosity function. This agrees well with the slopes found in other associations.

To construct the mass function we use two methods. First, we derive the masses of all main sequence stars in the CMD from the relation of $M_{V}$ and mass from the best fitting isochrone. A power law fit to the distribution yields a slope of $x=1.37 \pm 0.10$, equal to the Salpeter (1955) value. Second, we count all stars between the stellar evolutionary tracks in the HRD. Since for the conversion of the photometry to the theoretical plane the $U$ magnitude is essential, the completeness in the HRD is worse than in the CMD. Thus the count rates between the tracks in the HRD are smaller than in the comparable bins of the CMD. Surprisingly, the slope of the resulting mass function is nearly the same. A comparison of the mass function slope inside and outside the shell shows only a slightly steeper slope in the outside area. The slope we found for the IMF 
is very similar to the values found for other associations and open clusters. The idea of an universal shape of the IMF is thus again supported.

Acknowledgements. Many thanks go to Thomas Lehmann for providing his digital image for the correction of the shutter delay times and to Georges Meynet for the program to calculate isochrones from their tracks. We thank Hans-Joachim Tucholke for calculating the exact positions of our target stars for spectroscopy from the MACS and Klaas S. de Boer for a careful reading of the manuscript. We are indebted to You-Hua Chu for providing the photometry of Lee and to Sally Oey for providing us with digital versions of her photometry. We thank Kathy Eastwood for fruitful discussions. This research has made use of the Simbad database, operated at CDS, Strasbourg, France. JMW and DJB acknowledge support from the DFG Graduiertenkolleg "Magellanic Clouds". DJB is grateful for a FeodorLynen-Fellowship of the Alexander von Humboldt-Foundation.

\section{References}

Chin Y.-N., 1995, Ph. D. thesis, University of Bonn

Chu Y.-H., Mac Low M.-M., 1990, ApJ 365, 510

Chu Y.-H., Mac Low M.-M., Garcia-Segura G., Wakker B., Kennicutt R.C., 1993, ApJ 414, 213

Cohen R.S., Dame T.M., Garay G., et al., 1988, ApJ 331, L95

Davies R.D., Elliot K.H., Meaburn J., 1976, Mem. Roy. Astron. Soc. 81,89

de Boer K.S., Tucholke H.-J., Seitter W.C., 1995, ESO Messenger 81, 20

Efremov Y.N., 1988, Sov. Sci. Rev. E. Astrophys. Space Phys. 7,105

Elliot K.H., Goudis C., Meaburn J., Pilkington J., 1978, Ap\&SS 55, 475

Fitzpatrick E.L., 1991, PASP 103, 1123

Garmany C.D., Massey P., Parker J.Wm., 1994, AJ 108, 1256

Grothues H.-G., Gochermann J., 1992, ESO Messenger 68, 43

Heber U., Hunger K., Jonas G., Kudritzki R.P., 1984, A\&A 130, 119

Henize K.G., 1956, ApJS 2, 315

Hill R.J., Madore B.F., Freedman W.L., 1994, ApJ 429, 204

Hill V., Andrievsky S., Spite M., 1995, A\&A 293, 347

Howarth I.D., 1983, MNRAS 203, 301

Kontizas M., Maravelias S.E., Kontizas E., Dapergolas A., Bellas-Velidis I., 1996, A\&A 308, 40

Kurucz R.L., 1992, Model Atmospheres for Population Synthesis. In: Barbuy B., Renzini A. (eds.) Proc. IAU Symp. 149, The Stellar Populations of Galaxies. Kluwer, Dordrecht, p. 225
Landolt A.U., 1992, AJ 104, 340

Lee M.G., 1990, Ph.D. thesis, University of Washington

Lucke P.B., Hodge P.W., 1970, AJ 75, 171

Luks T., Rohlfs K., 1992, A\&A 263, 41

Magnier E.A., Chu Y.-H., Points S.D., Hwang U., Smith R.C., 1996, ApJ 464, 829

Malumuth E.M., Heap S.R., 1994, AJ 107, 1054

Malumuth E.M., Waller W.H., Parker J. Wm., 1996, AJ 111, 1128

Massey P., 1985, PASP 97, 5

Massey P., Johnson K.E., DeGioia-Eastwood K., 1995a, ApJ 454,151

Massey P., Lang C.C., DeGioia-Eastwood K., Garmany C.D., 1995b, ApJ 438, 188

McKibben Nail V., Shapley H., 1953, Proc. Nat. Acad. Sci. 39, 358

Meaburn J., Laspias V.N., 1991, A\&A 245, 635

Miller G.E., Scalo J.M., 1979, ApJS 41, 513

Mochizuki K., Nakagawa T., Doi Y., et al., 1994, ApJ 430, L37

Oestreicher M.O., Gochermann J., Schmidt-Kaler T., 1995, A\&AS 112, 495

Oey M.S., Massey P., 1995, ApJ 452, 210

Pakull M.W., Motch C., 1989, Nat 337, 337

Parker J.Wm., Garmany C.D., Massey P., Walborn N.R., 1992, AJ 103, 1205

Rogers F.J., Iglesias C.A., 1992, ApJS 79, 507

Salpeter E.E., 1955, ApJ 121, 161

Savage B.D., Mathis J.S., 1979, ARA\&A 17, 73

Schaerer D., Meynet G., Maeder A., Schaller G., 1993, A\&AS 98,523

Schmidt-Kaler T., 1982, in: Landolt-Börnstein, Neue Serie VI/2b. Springer, Berlin, 453-455 pp. 15-18,

Schwering P.B.W., Israel F.P., 1991, A\&A 246, 231

Spite F., Barbuy M., Spite M., 1993, A\&A 272, 116

Stasińska G., Testor G., Heydari-Malayeri M., 1986, A\&A 170, L4

Tucholke H.-J., de Boer K.S., Seitter W.C., 1996, A\&AS 119, 91

Vázquez R.A., Will J.-M., Prado P., Feinstein A., 1995, A\&AS 111,85

Walborn N.R., Fitzpatrick E.L., 1990, PASP 102, 379

Wang Q., Helfand D.J., 1991, ApJ 373, 497

Westerlund B.E., 1990, A\&AR 2, 29

Will J.-M., Bomans D.J., de Boer K.S., 1995a, A\&A 295, 54

Will J.-M., Vázquez R.A., Feinstein A., Seggewiss W., 1995b, A\&A 301, 396

Will J.-M., Bomans D.J., Vallenari A., Schmidt J.H.K., de Boer K.S., 1996, A\&A 315, 125 